\title{
A comparative analysis of the outer-belt primitive families
}

\author{
M. N. De Prá ${ }^{1}$, N. Pinilla-Alonso ${ }^{1}$, J. Carvano ${ }^{5}$, J. Licandro ${ }^{2,3}$, D. Morate ${ }^{5}$, V. Lorenzi ${ }^{4,2}$, J. de León ${ }^{2,3}$, \\ H. Campins ${ }^{6}$, and T. Mothé-Diniz ${ }^{7}$ \\ ${ }^{1}$ Florida Space Institute, University of Central Florida, Florida, USA \\ e-mail: mariodepra@ucf.edu \\ 2 Instituto de Astrofísica de Canarias, C/Vía Láctea s/n, 38205 La Laguna, Spain \\ ${ }^{3}$ Universidad de La Laguna (ULL), 38205 La Laguna, Spain \\ ${ }^{4}$ Fundación Galileo Galilei - INAF, Rambla José Ana Fernández Pérez, 7, 38712 Breña Baja, Santa Cruz de Tenerife, Spain \\ ${ }^{5}$ Departamento de Astrofísica, Observatório Nacional, Rio de Janeiro 20921-400, Brazil \\ ${ }^{6}$ Department of Physics, University of Central Florida, Florida, USA \\ ${ }^{7}$ Faculty of Natural Sciences, Norwegian University of Science and Technology (NTNU), 7491 Trondheim, Norway
}

Received 30 May 2020 / Accepted 24 August 2020

\begin{abstract}
Context. Asteroid families are witnesses to the intense collisional evolution that occurred on the asteroid belt. The study of the physical properties of family members can provide important information about the state of differentiation of the parent body and provide insights into how these objects were formed. Several of these asteroid families identified across the main belt are dominated by lowalbedo, primitive asteroids. These objects are important for the study of Solar System formation because they were subject to weaker thermophysical processing and provide information about the early conditions of our planetary system.

Aims. We aim to study the diversity of physical properties among the Themis, Hygiea, Ursula, Veritas, and Lixiaohua families.

Methods. We present new spectroscopic data, combined with a comprehensive analysis using a variety of data available in the literature, such as albedo and rotational properties.

Results. Our results show that Themis and Hygiea families, the largest families in the region, present similar levels of hydration. Ursula and Lixiaohua families are redder in comparison to the others and present no sign of hydrated members based on the analysis of visible spectra. Conversely, Veritas presents the highest fraction of hydrated members.

Conclusions. This work demonstrates a diverse scenario in terms of the physical properties of primitive outer-belt families, which could be associated with dynamical mixing of asteroid populations and the level of differentiation of the parental body.
\end{abstract}

Key words. minor planets, asteroids: general

\section{Introduction}

Asteroid families are remnants from the main-belt collisional history, and are identified by clusters in the proper orbital parameters (Hirayama 1918; Zappala \& Cellino 1992; Nesvorný et al. 2015). Members of an asteroid family consist of fragments of a parent body that were disrupted after experiencing an energetic collision in the past. The study of physical properties among family members, such as composition, degree of aqueous alteration, and spectral diversity, can provide important information about the state of differentiation of the parent body (Cellino et al. 2002). Knowledge of the internal structure of asteroids is particularly difficult to obtain, yet is critical to understand how, when, and where these objects were formed and to shed light on the formation and evolutionary history of the Solar System.

Several asteroid families identified across the main belt are dominated by low-albedo, primitive asteroids (Nesvorný et al. 2015; Nesvorny 2015). These objects are of particular importance to the study of the formation of the Solar System because they were subject to low thermophysical processing or none at all, and can provide information about the early conditions of our planetary system. Furthermore, investigations of the surface composition of these objects show that they can contain hydrated minerals, water ice, and organic materials (Vilas 1994; Fornasier et al. 2014; Campins et al. 2010; Rivkin \& Emery 2010; Licandro et al. 2011; Takir \& Emery 2012; Rivkin et al. 2015, 2019). These discoveries corroborate the hypothesis that primitive asteroids are a potential source of Earth's water and organic material (Morbidelli et al. 2000).

Advances in the characterization of primitive asteroids show that they present almost featureless visible and near-infrared (NIR) spectra, with a variety of surface colors from blue to red (for a review see DeMeo et al. 2015). A common feature observed in several of these objects across the asteroid belt is the $0.7 \mu \mathrm{m}$ absorption band, characteristic of hydrated minerals (Vilas 1994; Carvano et al. 2003; Fornasier et al. 2014). The number of objects presenting this feature peaks at the central belt and decreases at the Cybele region, delimiting the hydration zone (Rivkin 2012; Fornasier et al. 2014; De Prá et al. 2018a). As part of the PRIMitive asteroid spectroscopic survey (PRIMASS), we studied the visible spectroscopy of eight primitive asteroids families in the inner belt and identified a mix of anhydrous and hydrated families in that region, where the percentage of hydrated members in a family can reach nearly $80 \%$ (de León et al. 2016; Morate et al. 2016, 2018, 2019).

Primitive asteroid families are observed across the whole main belt, and they become the dominant population in the outer belt where most of their mass is contained (DeMeo \& Carry 2014). Nesvorný et al. (2015) identified nine large $(N>300$ members) primitive families in this region (Fig. 1): Themis, 


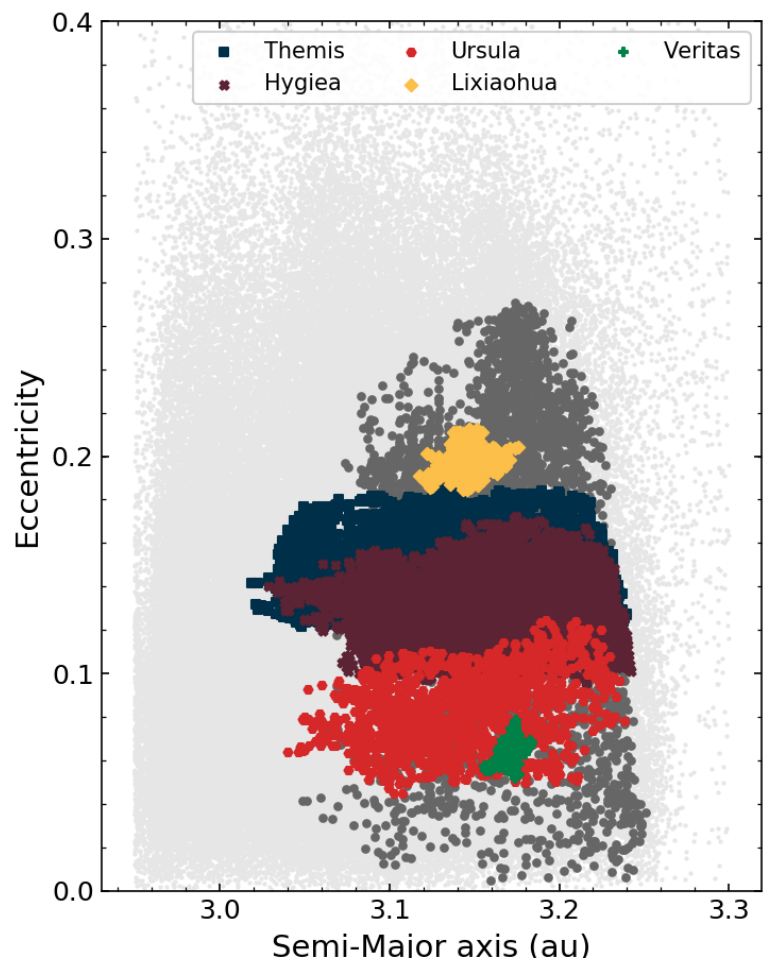

Fig. 1. Orbital properties of large outer-belt asteroid families. The studied families are shown in colors, while those that are not studied in this work are represented by dark gray dots. The light gray dots represent the background.

Hygiea, Ursula, Veritas, Lixiaohua, Alauda, Theobalda, Euphrosine, and Meliboea.

The first detection of water ice in the asteroid belt was made on (24) Themis, the largest member of its homonymous family (Campins et al. 2010; Rivkin \& Emery 2010). Recent works proposed that water ice is also present in large outer-belt asteroids (Takir \& Emery 2012; Rivkin et al. 2019). Additionally, the first observed main-belt comet (MBC), an active asteroid with proposed sublimation-driven activity, is also a member of the Themis family (Hsieh et al. 2004; Hsieh \& Jewitt 2006). Hsieh et al. (2018) proposed that there are seven MBCs which are linked to four families in outer belt: Themis, Lixiahoua, Alauda, and Theobalda.

The Themis family is one of the most highly studied families in the literature, in which both water ice and hydrated minerals have been observed among family members. Castillo-Rogez \& Schmidt (2010) and Marsset et al. (2016) interpreted these results as the product of a partial differentiation of the Themis parent body. The spectroscopic properties of the Lixiaohua family were studied in De Prá et al. (2020), and although it is also a MBCbearing family, it was found to present distinct properties from the Themis group, which can be interpreted as a difference in composition and/or differentiation level.

The aforementioned studies of outer-belt objects suggest that the hydrated to icy transition is likely to occur at the outer belt. In this work, we performed a comparative study of the physical properties among the outer-belt families by means of new spectroscopic data combined with data gathered from public catalogs. In Sect. 2 we describe the spectroscopic observations and data-reduction techniques. The analysis of these data is presented in Sect. 3, and we include the data available from public catalogs to extend the analysis to study the albedo, parent body size, and rotational properties of these families. The results for each family are presented in Sect. 4. In Sect. 5, we compare results across the families and discuss the implications of these findings.

\section{Observations and data reduction}

We collected low-resolution spectra for 29 asteroid members of the Themis, Hygiea, and Veritas families (Table A.1, Figs. A.1-A.3). The data were obtained using the Goodman High-Throughput Spectrograph (GTHS) at the 4.1m SOAR telescope on Cerro-Pachón, Chile. We used a setup with grating of 300 lines $/ \mathrm{mm}$ and the slits of $1.03^{\prime \prime}$ in 2011, and $1.68^{\prime \prime}$ in 2012 with no second-order blocking filter, which provided an effective spectral interval of $0.4-0.87 \mu \mathrm{m}$. Observations were made on a total of six nights across the semesters of 2011 A and 2012B. We also obtained two sequences of calibration quartz lamps immediately before and after target acquisition in order to reduce systematic errors. At least one solar analog was observed at different air masses during each night.

We used quartz lamps for the flat-field correction of the images, while the HgAr lamps were used for the wavelength calibration. The reduction was made using standard techniques: images were bias- and flat-field corrected and the spectra were extracted, background subtracted, and wavelength calibrated in sequence. This procedure was repeated for the three subexposures, and then averaged to produce a final spectrum for each target.

To obtain asteroid reflectance spectra, we divided the object spectrum by the spectra of solar analogs. We analyzed the spectra of the solar analog stars to detect small differences in color introduced during the observations, caused for example by inconsistent centering of the star in the slit. These differences could propagate into the reflectance spectrum of the target through the reduction process. The first step was to apply an atmospheric extinction correction. This effect is dependent on the airmass and the wavelength, with shorter wavelengths and higher air masses experiencing greater extinction. To minimize the extinction effect from the difference in airmass between the stars and the target, we applied a correction to the spectra of the object and the stars. In the absence of extinction coefficients for Cerro Pachón, we used the mean extinction coefficients for La Silla, because this observatory is located relatively close and at similar altitude to Cerro Pachón. A study of the variation of extinction coefficients from different sites suggested it is mostly influenced by the altitude of the site. For each night, we divided all of the spectra of the solar analogs by one used as reference, typically the one at lower airmass. Ideally, the result of this division should be a slopeless flat spectrum, with a normalized flux value of around 1. The star observations that appeared to present outliers (i.e., an uncommon shape relative to the others and/or spectral inclination variation above $5 \sigma$ ) were discarded and the asteroid reflectance spectra were obtained using the remaining stars. Finally, all reflectance spectra were normalized to 1 at $0.55 \mu \mathrm{m}$.

The data reduction was made by combining scientific Python libraries (Van Der Walt et al. 2011; Jones et al. 2001; Astropy Collaboration 2013, 2018; Hunter 2007) with IRAF $^{1}$ tasks, called through the PyRAF ${ }^{2}$ library.

\footnotetext{
1 IRAF is distributed by the National Optical Astronomy Observatories, which are operated by the Association of Universities for Research in Astronomy, Inc., under cooperative agreement with the National Science Foundation.

2 PyRAF is a product of the Space Telescope Science Institute, which is operated by AURA for NASA.
} 
M. N. De Prá et al.: A comparative analysis of the outer-belt primitive families

Table 1. Outer belt families identified by Nesvorný et al. (2015) and studied in this work.

\begin{tabular}{|c|c|c|c|c|c|c|c|c|c|c|}
\hline $\begin{array}{l}\text { Asteroid } \\
\text { family }\end{array}$ & $\begin{array}{c}a \\
(\mathrm{au})\end{array}$ & $e$ & $\begin{array}{c}i \\
\left({ }^{\circ}\right) \\
\end{array}$ & $\begin{array}{l}\text { Age } \\
(\mathrm{Gyr})\end{array}$ & $\begin{array}{c}\text { Members } \\
\left(\mathrm{N}^{\circ}\right)\end{array}$ & $\begin{array}{c}\text { Spectra } \\
\left(\mathrm{N}^{\circ}\right)\end{array}$ & $\begin{array}{c}\text { SDSS } \\
\left(\mathrm{N}^{\circ}\right)\end{array}$ & $\begin{array}{c}\text { MOVIS } \\
\left(\mathrm{N}^{\circ}\right)\end{array}$ & $\begin{array}{c}\text { WISE } \\
\left(\mathrm{N}^{\circ}\right)\end{array}$ & $\begin{array}{c}\text { ALCDEF } \\
\left(\mathrm{N}^{\circ}\right) \\
\end{array}$ \\
\hline Themis & 3.135 & 0.153 & 10.149 & $2.5 \pm 1.0$ & 4782 & 59 & 774 & 284 & 2312 & 443 \\
\hline Hygiea & 3.142 & 0.136 & 1.084 & $2.0 \pm 1.0$ & 4854 & 25 & 783 & 182 & 2234 & 149 \\
\hline Veritas & 3.174 & 0.066 & 5.103 & $0.0083 \pm 0.0005$ & 1294 & 17 & 234 & 34 & 715 & 41 \\
\hline Ursula & 3.129 & 0.092 & 9.164 & $<3.5$ & 1466 & 7 & 266 & 71 & 957 & 70 \\
\hline Lixiaohua & 3.153 & 0.197 & 3.215 & $0.15 \pm 0.05$ & 756 & 35 & 110 & 24 & 514 & 28 \\
\hline
\end{tabular}

Notes. See text for details.

\section{Analysis}

In addition to the 29 spectra observed, we collected all available spectra in the literature of members of Themis, Hygiea, Veritas, Ursula, and Lixiaohua families (Xu et al. 1996; Bus \& Binzel 2004; Vilas et al. 2006; Lazzaro et al. 2007; De Prá et al. 2020). In particular, the spectroscopic analysis of the Lixiaohua was recently presented in De Prá et al. (2020), in which we used the same methodology described in this section. Therefore, the individual analyses of each spectrum of the Lixiaohua family are not presented in this paper. In addition to the spectroscopic characterization, we include the albedo, diameters, visible and NIR colors, and rotational periods of each family. Table 1 includes the orbital parameters of the largest member of each family, the family age from Brož et al. (2013), the number of members with observed spectra (both observed in this work and taken from the literature), members with SDSS colors (Carvano et al. 2010; Hasselmann et al. 2011), NIR MOVIS colors (Popescu et al. 2016), WISE albedos and diameters (Mainzer et al. 2016), and rotational properties with $U>2$ (Warner et al. 2009).

\subsection{Spectroscopic analysis}

The entire analysis of the spectroscopic data was performed using the CANA package (De Prá et al. 2018b) ${ }^{3}$. In order to analize the spectra, we performed a taxonomical classification, and we also computed (whenever the wavelength coverage allowed it) four different parameters. These parameters are: visible slope (visible - red-mode), NIR slope (NIR), hydration feature (visible - red-mode), and turn-off point (visible - blue-mode).

The visible slope was measured using the angular coefficient in a linear fit to the spectrum in the wavelength range of 0.5 $0.9 \mu \mathrm{m}$, normalized to $0.55 \mu \mathrm{m}$. The spectral slopes are expressed in units of $\% / 1000 \AA$. The associated error is the sum of the systematic error introduced by the solar analogs division, and the error of the fit estimated by a Monte-Carlo method with 1000 iterations, where the spectra reflectance was resampled considering the spectrum S/N. Although the results presented are the quadratic sum of the described error sources, the systematic error is the strongly dominating term.

We looked for the presence of hydrated minerals through the identification and characterization of an absorption band centered at around $0.7 \mu \mathrm{m}$. For this task we used the wavelength range of $0.55-0.88 \mu \mathrm{m}$. The spectral continuum was estimated by a linear fit within the $0.55-0.57$ and $0.84-0.88 \mu \mathrm{m}$ intervals. Thereafter, we removed the continuum from the spectrum and fit a fourth-order spline in the $0.55-0.88 \mu \mathrm{m}$ range. We identified the hydration band if the minimum reflectance of the fit was close to $0.7 \mu \mathrm{m}$, at a depth greater than $1 \%$ and of $3 \sigma$ of the spectrum $\mathrm{S} / \mathrm{N}$.

\footnotetext{
3 CANA code is available though https://cana. readthedocs.io
}

A common feature in primitive asteroids is a turn-off in the slope at approximately $0.5 \mu \mathrm{m}$. We characterized the presence of this feature using the data available in the $0.4-0.7 \mu \mathrm{m}$ range, applying the same methodology as described in De Prá et al. (2018a).

Taxonomic classification of the asteroids was carried out by minimization of the chi-squared between the spectrum of a given object and the templates from the DeMeo et al. (2009) taxonomic system. This procedure was performed using the combined spectra with data from all wavelength ranges available. If the spectrum was sampled only at visible wavelengths, we adjusted the class to fit the Bus \& Binzel (2002) scheme. The results for the spectroscopic analysis are shown in Table A.2.

\subsection{Public photometric catalogs}

In addition to the spectroscopic data, we collected data of the families available from public databases. Visible colors were extracted from the SDSS (Carvano et al. 2010; Hasselmann et al. 2011), NIR color from the MOVIS catalog (Popescu et al. 2016), and geometric albedos from the NEOWISE survey (Mainzer et al. 2016).

We calculated the visible and NIR spectral slopes from the data available in these databases such that they are comparable with the ones derived from the spectroscopic data. We estimated the angular coefficient from a linear fit using the reflectances on $g, r$, and $i$ SDSS filters, normalized at $0.55 \mu \mathrm{m}$ for the visible slope; and using the $Y, H$, and $K$ VISTA filters, normalized in $1.2 \mu \mathrm{m}$ for the NIR slope. The criteria for the choices of filters was based on selecting the highest number of objects that were observed in at least three filters and covering almost the same wavelength range as the spectroscopic data (0.5-0.9 and $1.0-2.3 \mu \mathrm{m})$. To estimate the uncertainties, we created 1000 clones of each observation by drawing random values for the reflectance in each filter using normal distributions with means equal to the listed reflectance value and variances equal to the listed uncertainties. The resultant spectral slope distribution was then fitted with a Gaussian curve, the mean and variance of which were then adopted as the nominal value for the spectral slope and its uncertainty, respectively, expressed in units of $\% / 1000 \AA$. Finally, the mean visible and NIR slopes of each family were then estimated by a weighted average, using the slope uncertainties as the weights. A weighted average, with the uncertainties provided by the NEOWISE catalog, was used to estimate the mean albedo for each family. The results of this analysis are shown in Fig. 2 and Table 2.

\subsection{Parent body sizes}

We estimated the parent body diameter (PB) for each family based on the work of Brož \& Vokrouhlický (2008). To build 

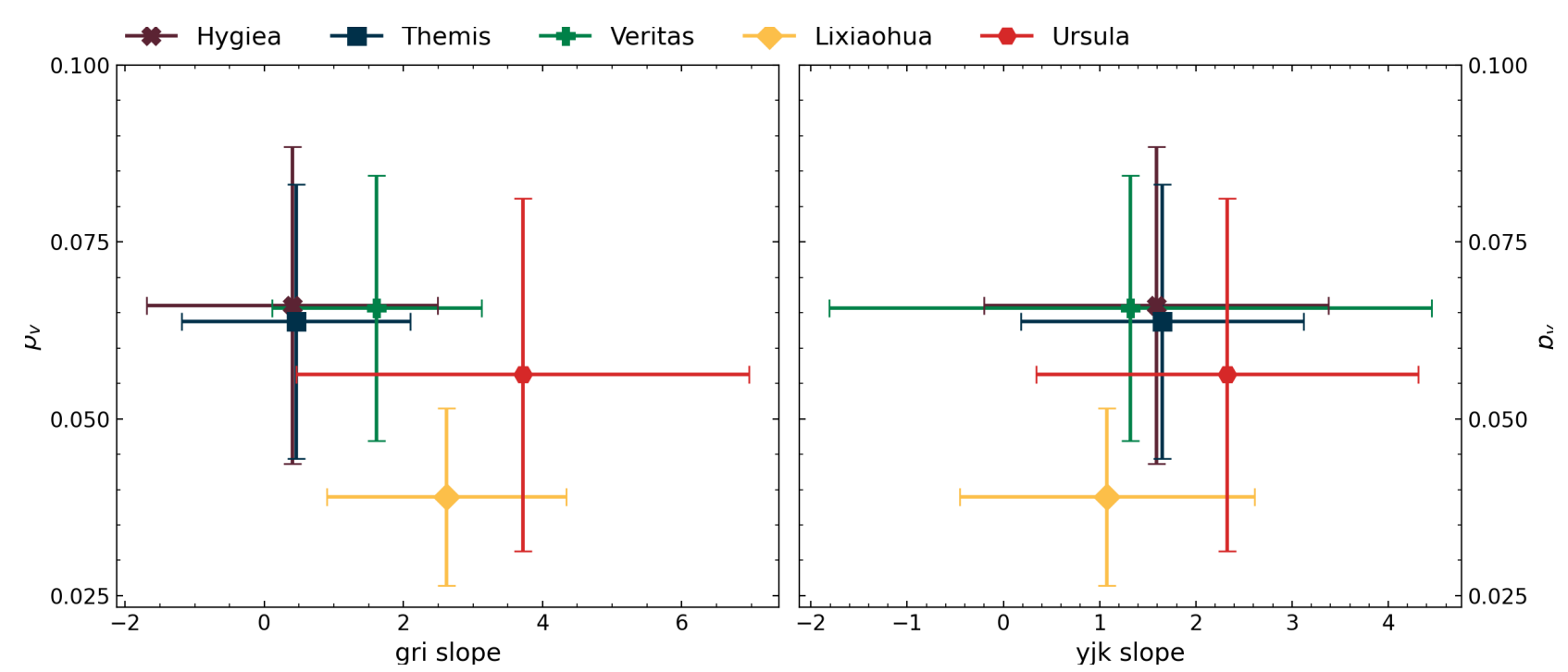

Fig. 2. Weighted average visible slope (left) and NIR slope (right) vs. albedo for five outer-belt asteroid families.

Table 2. Average physical properties of outer-belt families.

\begin{tabular}{|c|c|c|c|c|c|c|c|c|c|c|c|c|}
\hline $\begin{array}{l}\text { Asteroid } \\
\text { family }\end{array}$ & $\begin{array}{c}\text { Vis. slope } \\
\text { spec. } \\
(\% / 1000 \AA)\end{array}$ & $\begin{array}{l}\text { Vis. slope } \\
\text { SDSS } \\
(\% / 1000 \AA) \\
\end{array}$ & $\begin{array}{c}\text { IR slope } \\
\text { MOVIS } \\
(\% / 1000 \AA) \\
\end{array}$ & $\begin{array}{c}\text { Turn-off } \\
\text { members } \\
\text { Nf (Ns) }\end{array}$ & $\begin{array}{c}\text { Hydrated } \\
\text { members } \\
\mathrm{Nf}(\mathrm{Ns})\end{array}$ & $\begin{array}{c}\text { 3-micron } \\
\text { feature } \\
\text { (shape) }\end{array}$ & $\begin{array}{c}\text { MBC } \\
\text { members } \\
\text { (number) }\end{array}$ & Albedo & $\begin{array}{l}\text { PB } \\
\text { TW } \\
(\mathrm{km}) \\
\end{array}$ & $\begin{array}{c}\text { PB } \\
\text { Durda07 } \\
(\mathrm{km}) \\
\end{array}$ & $\begin{array}{c}\mathrm{LM} / \mathrm{PB} \\
\mathrm{TW}\end{array}$ & Density \\
\hline Themis & $-0.2 \pm 1.6$ & $0.5 \pm 1.6$ & $1.7 \pm 1.5$ & $11(21)$ & $8(59)$ & Rounded & 3 & $0.066 \pm 0.033$ & 286 & 451 & 0.68 & 1.2 \\
\hline Hygie & $0.3 \pm 3.7$ & $0.4 \pm$ & 1.6 & $10(13)$ & $3(25)$ & Ceres/rounded & 0 & $0.068 \pm 0.042$ & 492 & 442 & 0.88 & 1.5 \\
\hline Veritas & $1.0 \pm 1.3$ & $1.6 \pm$ & $1.3 \pm$ & $14(15)$ & $13(17)$ & - & 0 & $0.068 \pm 0.062$ & 135 & 177 & 0.88 & - \\
\hline Ursula & & & & & & Rounded & 0 & & 226 & 280 & 0.83 & 0.8 \\
\hline Lixiaohua & $3.0 \pm 3.2$ & $2.8 \pm 1.7$ & $1.1 \pm 1.5$ & $5(30)$ & $0(35)$ & - & 2 & $0.043 \pm 0.081$ & 69 & 220 & 0.51 & - \\
\hline
\end{tabular}

Notes. We present values for parent-body diameters (PB) obtained in this work (TW) and by Durda et al. (2007) (Durda07) for comparison. The ratio between the diameter of the families' largest member and that of the parent body (LM/PB) are shown only for diameters obtained in this work. Nf represents the number of objects that present the feature, while Ns is the number of spectra for which the wavelength was appropriate to perform the feature analysis.

the size-frequency distribution (SFD), we obtained diameters of the family members from the WISE survey (Mainzer et al. 2016). Objects with a geometric albedo $p_{v}>0.12$ were removed in order to consider only objects that could have a primitive surface and minimize the presence of interlopers. For the objects that did not have a determined diameter, we estimated it using the following equation:

$D=\frac{1329 \times 10^{-0.2 H}}{\sqrt{p_{\text {fam }}}}$,

where $H$ is the absolute magnitude of the object and $p_{\text {fam }}$ is the mean geometric albedo of the respective family. Figure 3 shows the SFD for each family, where it is possible to observe that there is an inflection point in the diameter distribution towards the smaller objects. We consider, as an approximation, that this inflection is related to an observational bias and use these points as the cutoff in diameter $\left(D_{c}\right)$ to define the sample completeness. The volume of the $\mathrm{PB}\left(V_{\mathrm{PB}}\right)$ can be estimated using:

$V_{\mathrm{PB}}=V_{c}+\int_{0}^{D_{c}}\left(\alpha \log _{10}(D)+\beta\right) \mathrm{d} D$,

where $V_{c}$ is the sum of volumes of the objects with $D>D_{c}$. The second term is the integral of a power-law fitted considering $D>D_{c}$, where $\alpha$ and $\beta$ are the coefficients of the fit. The integral limits are set from 0 to $D_{c}$ in order to add the contribution of the small-sized members. Finally, the diameter of $\mathrm{PB}\left(D_{\mathrm{PB}}\right)$ is estimated assuming a spheroidal shape:

$D_{\mathrm{PB}}=\sqrt[3]{\frac{6}{\pi} V_{\mathrm{PB}}}$

Figure 3 shows that, with the exception of Hygiea, the SFDs for all famailies are well approximated by a power law in the regime of $D>D_{c}$. In the case of Hygiea, we also defined an upper limit in diameter to constrain the region where the power law was fitted.

Although we use the power-law fitting to minimize the errors on the parental body size derived from the observational bias on smaller members, there are several sources of error in this estimate. For example, the individual errors in the diameter of the family members available in the WISE catalog are not considered here. Additionally, family members can be removed from the region, for example through Yarkovsky-driven evolution or chaotic diffusion by crossing resonances with giant planets. The process followed to identify families considers a cutoff in velocity, which typically leaves a halo of potential family members with similar surface properties, but with slightly distinct orbital parameters that are not considered as belonging to the families (Nesvorný et al. 2015). Finally, even though we defined a range in geometric albedo to minimize the presence of interlopers, there 


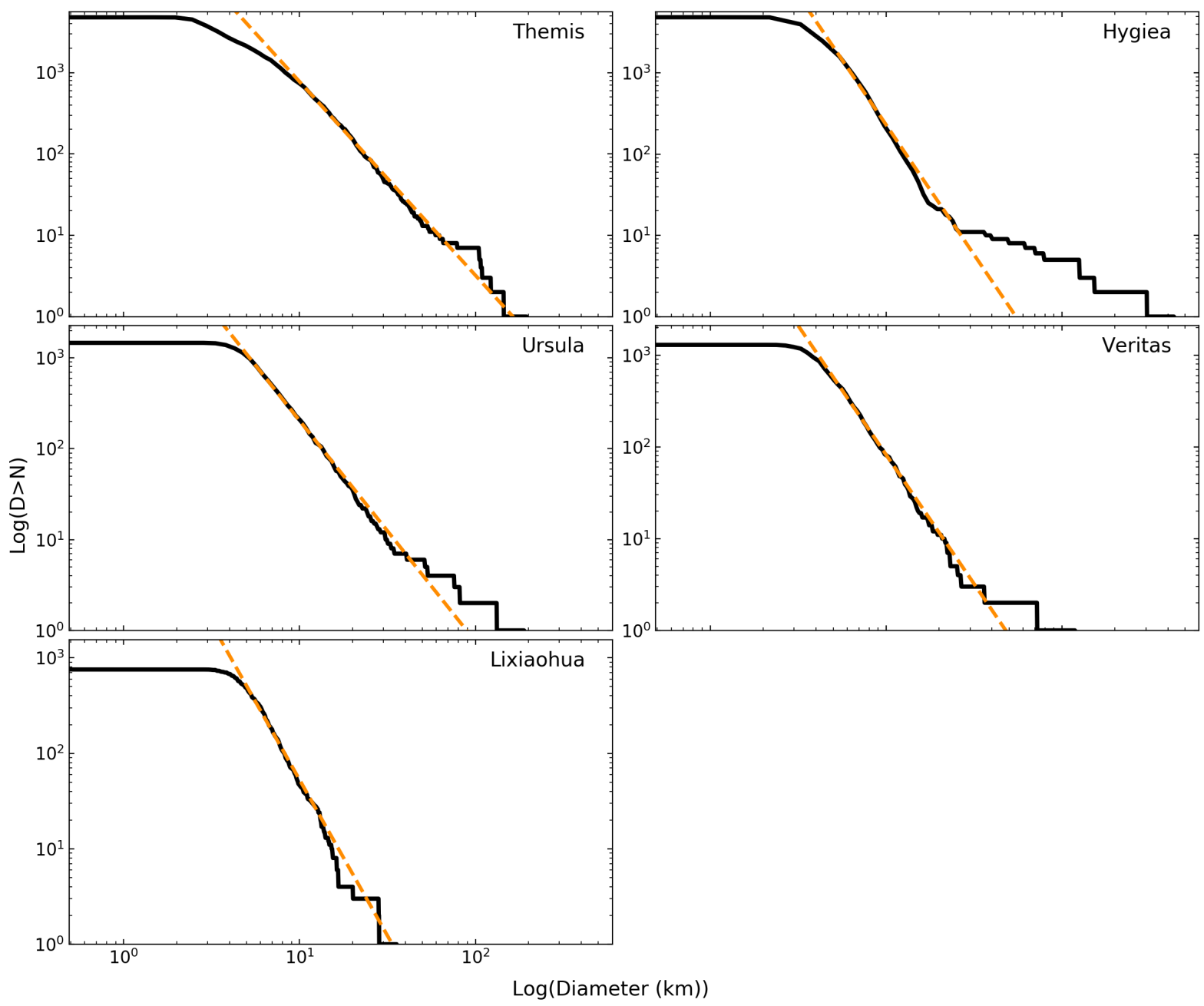

Fig. 3. SFD for the five outer-belt asteroid family. The orange line represents the power-law fit that was used to include the contribution of small-sized members into the calculation of the family's parent body size.

should still be objects that are not true members of the family but that occupy the same region and may have been added in the SFD. The procedure of selecting only the family members with albedo $p_{v}>0.12$ revealed that 1 to $12 \%$ of the family members can be outliers.

\subsection{Density}

The analysis of asteroid rotational properties performed by Pravec \& Harris (2000) showed that objects larger than $0.15 \mathrm{~km}$ do not rotate faster than 11 revolutions per day. This spin barrier is interpreted by means of a rubble-pile structure model, in which rotation with periods exceeding the critical value would cause asteroid breakup (Pravec \& Harris 2007). The smaller objects with faster rotation are thought to be monolithic collisional fragments with stronger cohesion forces.

In a cohesionless rubble pile, the critical value for the spin barrier is dependent on bulk density (Eq. (4)). The bulk density of a body depends on both the grain density of the material and the total porosity in the body. The grain density is related to the composition, while the porosity is linked mostly to the collisional history of the body. Asteroid families with distinct formation histories, composition, and differentiation level should present differences in densities, which would be reflected by distinct spin barriers.
We performed an estimate of the density of the family members based on their rotational properties. This analysis considers two a priori hypotheses: (1) all objects with diameter within the 4-20 km range in a family that are close to the spin barrier have the same density, and (2) the original spin distribution reached the spin barrier, or the family has existed for a sufficiently long time for YORP effects to increase the spin up to the spin barrier. Considering this scenario and assuming that the structure of objects with diameters in the 4-20 km range can be approximated by a cohesionless rubble pile (Pravec \& Harris 2000), we can use the following equation to infer these properties:

$\rho \approx \frac{(1+\Delta m)}{\left(P_{\text {crit }} / 3.3\right)^{2}}$,

where $\rho$ is the bulk density, $P_{\text {crit }}$ is the critical value for the rotation period (in hours), and $\Delta m$ is the amplitude of the rotational light curve. For this analysis, we use a spherical shape approximation, where $\Delta m=0$.

To determine the critical value for each family, we searched for cataloged rotational periods of family members in the Asteroid Light curve DataBase (LCDB), Updated 2019 August 14 (Warner et al. 2009), with the U parameter of the light curve equal to or higher than two. The U parameter is defined based on the quality and coverage of light curve used to estimate the 

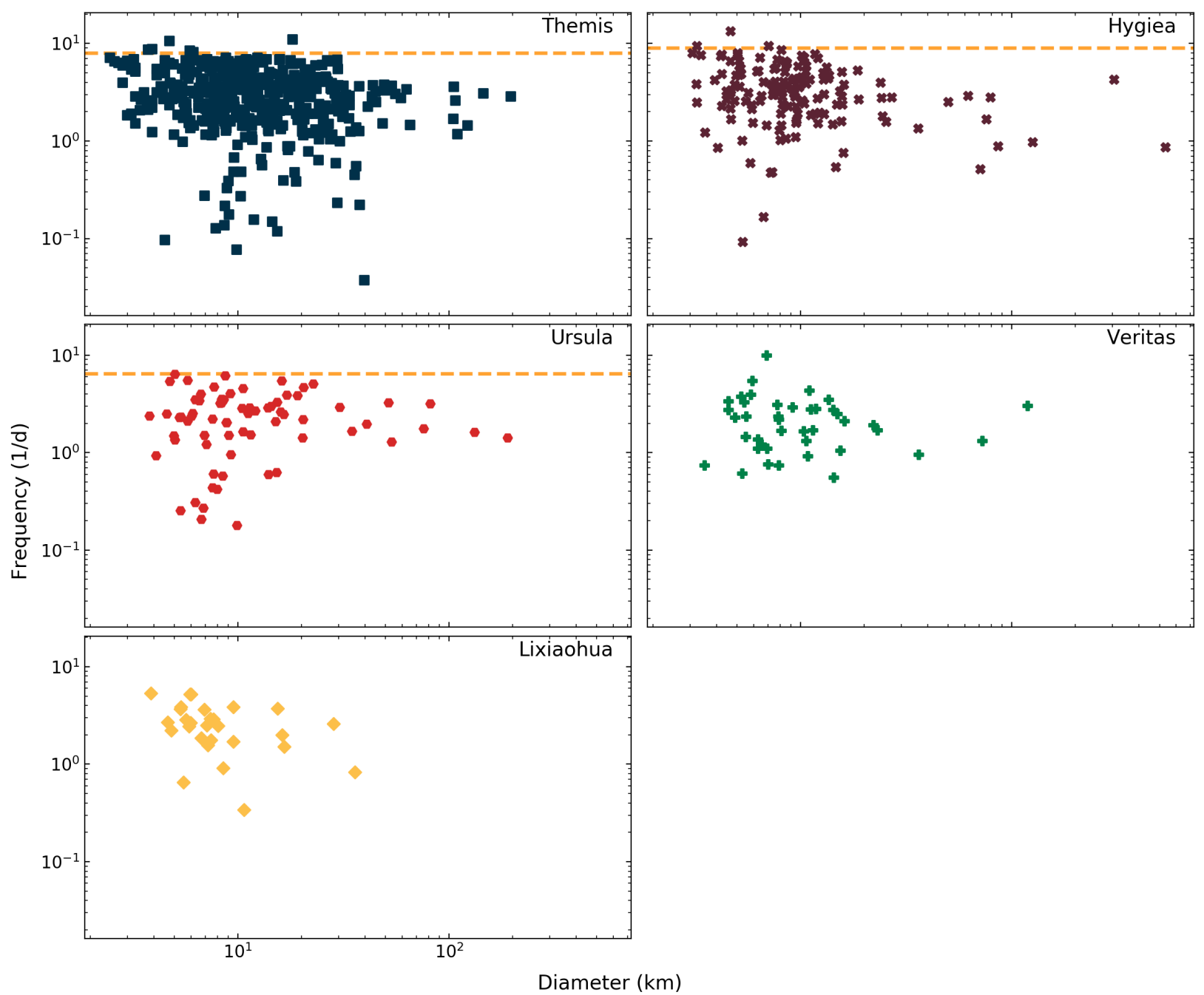

Fig. 4. Rotation frequency vs. diameter for each family. The orange line delimits the estimated critical period. For Veritas and Lixiaohua, the small number of determined periods prevents the determination of this value.

rotational period, a value of 2 is the recommended value for statistical analysis and indicates that the light curve was not completely observed and that the period uncertainty can reach up to $30 \%$. The number of objects per family that satisfies this criteria is shown in Table 1. Figure 4 shows the frequency versus diameter plot for each family with the respective critical period value.

We note that the number of cataloged rotational periods per family can be too small for the determination of a spin-barrier. This is seen especially in the case of the Lixiaohua and Veritas families. Additionally, these families possess the two youngest estimated ages in our sample (Table 1), and their spin distribution could be less affected by the YORP effect and collisional evolution, so much so that they do not yet present a spin barrier. We note that the estimated densities are the result of a tentative approach that will become more robust as we increase the number of determined rotation periods.

\section{Results}

\subsection{Themis}

We increased the number of members with observed spectra to reach a total of 59 family members by adding family data available in the literature (Florczak et al. 1999; Mothé-Diniz et al. 2005; Ziffer et al. 2011; Licandro et al. 2012; de León et al. 2012; Kaluna et al. 2016; Fornasier et al. 2016; Landsman et al. 2016; Marsset et al. 2016). The mean visible slope was estimated at $-0.17 \pm 1.58 \% / 1000 \AA$, and $13 \%$ of the sample showed the $0.7 \mu \mathrm{m}$ feature. The majority of the objects were classified as belonging to C-class, with a significant fraction of B-types, hence the negative mean slope. This value is consistent, considering the errors, with the $0.45 \pm 1.64 \% / 1000 \AA$ estimated using SDSS data for 563 objects. There were 21 out of the 59 spectra with appropriate wavelength coverage for the Turn-off analysis, 11 of which showed this feature. The critical wavelength for the Turn-off change can vary significantly from $0.49-0.67 \mu \mathrm{m}$. The MOVIS data provided a mean NIR slope of $1.65 \pm 1.47 \% / 1000 \AA$, slightly higher than the $0.12 \pm 0.07 \% / 1000 \AA$ obtained using spectroscopic data by Ziffer et al. (2011). The latter work used the wavelength range of $1.6-2.3 \mu \mathrm{m}$ to measure the slope, which is distinct from the $1.0-2.15 \mu \mathrm{m}$ provided by the MOVIS data, which was used in this work. Additionally, these latter authors found that many Themis family members can show a concave positive curvature in the spectra. Both factors could be influencing the slope determination from the photometric data. 
The Themis parent body size was estimated to be approximately $286 \mathrm{~km}$, in agreement with the value obtained by Brož et al. (2013), which was calculated in a similar manner. However, this value is considerably smaller than those obtained using different techniques by Durda et al. (2007) and Tanga et al. (1999), of 451 and $369 \mathrm{~km}$ respectively. Nonetheless, the estimated values of $\mathrm{PB}$ provide a range of $L M / P B$ (largest member diameter divided by the parent body size) from $0.51-0.68$, which is compatible with a catastrophic event being the origin for the family.

The analysis of the rotational properties of 443 Themis family members presented the most well-defined spin barrier among the studied families. The critical period at $\sim 3 \mathrm{~h}$ provides an estimate of the bulk density of $1.2 \mathrm{~g} \mathrm{~cm}^{-3}$. The bulk density of (24) Themis was estimated at $1.81 \pm 0.67 \mathrm{~g} \mathrm{~cm}^{-3}$ by Carry (2012), while Descamps et al. (2007) studied the binary asteroid (90) Antiope, a member of the Themis family, and found a density of $1.25 \pm 0.05 \mathrm{~g} \mathrm{~cm}^{-3}$. Both values are in agreement with the estimate in this work, accounting for the error bars. Another binary member of the family, (379) Huenna, was studied by Marchis et al. (2008), who found a bulk density of $0.9 \pm 0.1 \mathrm{~g} \mathrm{~cm}^{-3}$, slightly under the value estimated by the spin-barrier method. However, the diameter used to estimate (379) Huenna bulk density was higher than the one provided by Mainzer et al. (2016), and a smaller diameter would return a higher value for the density. Additionally, it is of note that larger objects $(D>30 \mathrm{~km})$ can follow a different regime in terms of cohesive forces and density, as proposed by Pravec \& Harris (2000).

\subsection{Hygiea}

The Hygiea family showed very similar properties to the Themis family at the studied wavelengths, with a mean slope of $0.30 \pm 3.68 \% / 1000 \AA$ (spectroscopic) and $0.40 \pm 2.09 \% /$ $1000 \AA$ (photometric) in visible wavelengths, and $1.07 \pm 1.53 \%$ / $1000 \AA$ in the NIR. However, there is a higher diversity in spectral slope than in Themis. Most of the targets were also classified as C-class, with a significant B-type contribution. Approximately $15 \%$ of the family members present the $0.7 \mu$ m hydration band, and 12 out of 17 objects show the turn-off feature.

Asteroid (10) Hygiea is the largest object in the outer-belt region, and the fourth largest in the main belt. The diameter cataloged by Mainzer et al. (2016) for (10) Hygiea is of $\sim 533 \mathrm{~km}$, considerably higher than the previous estimate from Tedesco (1994) of $\sim 407 \mathrm{~km}$. A recent work of Vernazza et al. (2019) using a direct imaging technique estimated an equivalent diameter of $\sim 434 \mathrm{~km}$. We adopted this latter value for the object and to construct the family SFD. The parental body size was estimated at $486 \mathrm{~km}$, with $L M / P B \sim 0.89$. Durda et al. (2007) estimated the family $\mathrm{PB}$ to be $440 \mathrm{~km}$, in good agreement considering that these latter authors used a different technique and a smaller diameter for (10) Hygiea. Figure 3 reveals an excess of large asteroids which is characteristic of a shattering event, where most of the family mass is distributed among the largest members. These values are influenced by the presence of other large asteroids besides (10) Hygiea, such as (52) Europa and (159) Aemilia, with respective diameters of $\sim 303 \mathrm{~km}$ and $\sim 125 \mathrm{~km}$. Both of these objects were identified as Hygiea family members by Nesvorný et al. (2015), but not in Vernazza et al. (2019) and Carruba (2013). The exclusion of these objects from the family would produce a smaller parent body, where the secondlargest body would be (1599) Giomus with a diameter of $52 \mathrm{~km}$. The presence of (52) and (159) can significantly change the interpretation of the collisional event that formed the family and of the nature of the family parent body.

A rotation period has been derived for 149 members of the Hygiea family. The spin barrier is defined at $\sim 2.7 \mathrm{~h}$, which provides a density estimate of $1.5 \mathrm{~g} \mathrm{~cm}^{-3}$. In Vernazza et al. (2019), the authors estimated a bulk density of $1.944 \mathrm{~g} \mathrm{~cm}^{-3}$ for (10) Hygiea, which is in reasonable agreement with the $2.19 \pm 0.42 \mathrm{~g} \mathrm{~cm}^{-3}$ calculated by Carry (2012). These values are considerably higher than our estimates. However, (10) Hygiea is considerably larger than the regime where we use the cohesionless approximation (4-20 km). Also, considering that this object could be a differentiated body, and could have different layers of material and levels of compaction, a higher value of this object density is plausible and not contradictory to the possibility that smaller objects could have lower densities. Carry (2012) also estimated a density of $1.52 \pm 0.39 \mathrm{~g} \mathrm{~cm}^{-3}$ for (52) Europa, which is in agreement with the value obtained in this work.

\subsection{Veritas}

The Veritas family showed the highest spectral homogeneity among its members, with a mean visible slope of $1.00 \pm 1.30 \% / 1000 \AA$ (spectroscopic) and $1.61 \pm 1.50 \% /$ $1000 \AA$ (photometric) and a high content of hydrated members reaching $77 \%$ of the sample. Of the 16 objects analyzed in this family, 14 show the Turn-off feature. The majority of the objects' spectra were classified in the C-complex, especially as Ch-type, which reflects the high level of hydrated members in the family. This result is in agreement with that of Mothé-Diniz et al. (2005). The mean NIR slope was estimated at $1.31 \pm 3.12 \% / 1000 \AA$, higher than the $-0.04 \pm 0.03 \% / 1000 \AA$ obtained using spectroscopic data obtained by Ziffer et al. (2011). These latter authors noted that, in contrast to the Themis family, the NIR spectra for the Veritas family show a concave negative curvature. As in the Themis family case, the wavelength range used to determine the slope and the curvature in the spectra could be influencing the different values obtained in this work.

The Veritas parental body size was estimated at $136 \mathrm{~km}$, which is slightly smaller than the $177 \mathrm{~km}$ obtained by Durda et al. (2007). Both values are in agreement with those of Brož et al. (2013), who estimated the Veritas PB at 100-177 km. The small number of cataloged rotational periods for Veritas members prevents us from obtaining an estimate of the family spin barrier and density limit.

\subsection{Ursula}

The Ursula family present a spectroscopic mean slope of $2.77 \pm 1.46 \% / 1000 \AA$, compatible with the $3.71 \pm 3.25 \%$ / $1000 \AA$ A estimated using SDSS data. Based on NIR colors, Ursula showed the reddest mean slope among the studied families. None of the objects exhibited the $0.7 \mu \mathrm{m}$ feature, indicating that the family might have a low hydrated mineral content. Although the spectra for (375) Ursula obtained by Bus \& Binzel (2004) and Vilas et al. (2006) presented similar spectral slopes, there is a significant difference in the shape of the spectra, and the data from the latter work might suggest a hydration feature.

We estimated the parent body diameter at $226 \mathrm{~km}$, which is slightly larger than the $203 \mathrm{~km}$ estimated by Brož et al. (2013) and smaller than the $280 \mathrm{~km}$ measured by Durda et al. (2007). The rotational distribution suggests a spin barrier with a critical rotational period of $\sim 3.6 \mathrm{~h}$, which provides an estimate for the density for the Ursula family of $\sim 0.8 \mathrm{~g} \mathrm{~cm}^{-3}$. 


\subsection{Lixiaohua}

The results for the spectroscopic analysis of the Lixiaohua family are described in De Prá et al. (2020), where we found a mean slope of $3.03 \pm 3.22 \% / 1000 \AA \quad$ (spectroscopic) and $2.79 \pm 1.72 \% / 1000 \AA$ (spectrophotometric) in visible wavelengths, and of $1.07 \pm 1.53 \% / 1000 \AA$ in the NIR. The Lixiaohua is the darkest of the studied families, with mean albedo of $0.043 \pm 0.081$ (Fig. 2).

In this work, we extended the analysis by calculating the parent body size, estimated at $P B \sim 69 \mathrm{~km}$ and the ratio $L M / P B \sim 0.51$, which are consistent with a catastrophic disruption event. This $\mathrm{PB}$ value is significantly smaller than the one estimated by Durda et al. (2007), of $\sim 220 \mathrm{~km}$, who also provide $L M / P B \sim 0.16$. The latter value suggests that the majority of the parent-body mass was scattered or lies in the smaller undiscovered family members. There are only 28 Lixiaohua members with determined rotational periods. The analysis of Fig. 4 suggests that the family spin barrier might be considerably low. However, more data are needed to obtain any conclusive density estimate.

\section{Discussion}

The analysis of the outer-belt families Themis, Hygiea, Veritas, Ursula, and Lixiaohua reveals a diversity of physical properties. In Table 2, we present the results for the spectroscopic properties, parent-body sizes, densities, and include information that contributes to this discussion: the $3 \mu \mathrm{m}$ band information extracted from the literature (Campins et al. 2010; Takir \& Emery 2012; Rivkin et al. 2019) and the presence of main-belt comets (Hsieh et al. 2018). In particular, the presence of hydrated and ice-bearing objects in a single family can be considered an indication of partial differentiation of the parental body, as proposed for the Themis family. Castillo-Rogez \& Schmidt (2010) and Marsset et al. (2016) suggested that these two materials could be representatives of different layers in the internal structure of the family's parent body. Hydrated minerals, which are typically formed by the interaction of liquid water with silicates such as olivine, would have formed in the inner layers that experienced higher temperature and pressure conditions, while the water ice could remain nearly unaltered in the external layers. In contrast, an undifferentiated body should present members with only hydrated minerals or water ice, but not both.

Figure 5 shows the median and mean slope of each family with associated variance and minimum and maximum values, while Fig. 2 shows the albedo visible and NIR slopes obtained from public catalogs. Themis, Hygiea, and Veritas families show neutral visible slopes compatible with C-type asteroids, with a higher variability in the Hygiea family. In contrast, Ursula and Lixiaohua show slightly reddish slopes, related to P-type objects.

We identified different hydrated fractions among the families. The two large C-type families Themis and Hygiea show a similar percentage of hydrated members at $13-15 \%$, while the smaller Veritas family shows a higher fraction of hydrated members, reaching $87 \%$. The Veritas family also shows the highest fraction of members showing the turn-off feature. This feature in C-type objects is attributed to ferric oxide intervalence charge transfer transition Feierberg et al. (1985); Vilas (1995). Hendrix \& Vilas (2019) proposed the absence of the feature could be related to a higher degree of space weathering. This result could explain why Themis is the oldest family in our sample and shows a lower fraction of turn-off members,

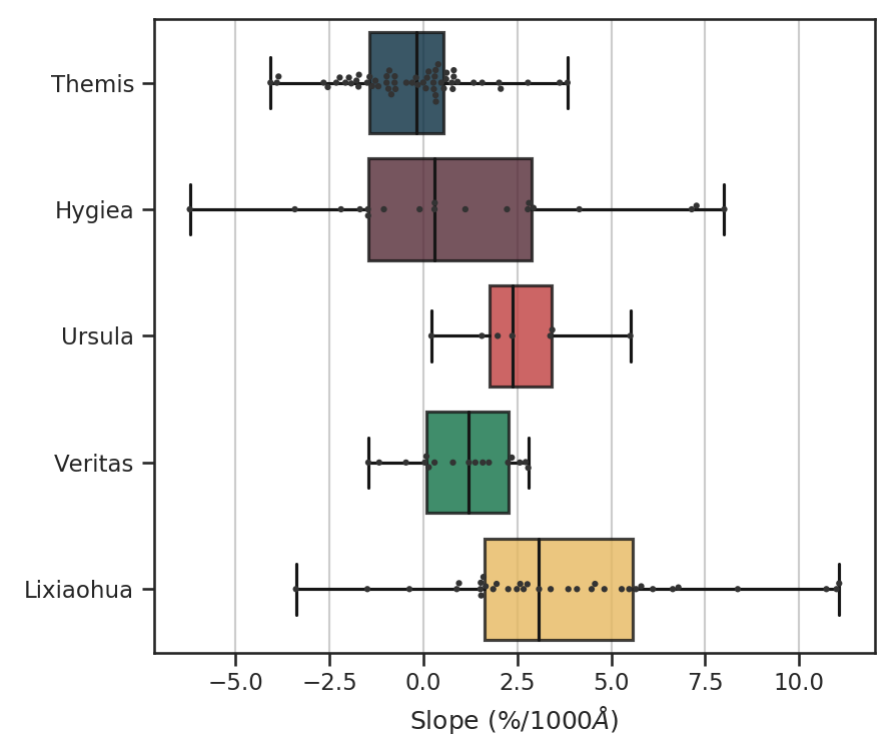

Fig. 5. Whisker plot of the visible slope distribution in each family. The boxes represent the average and standard deviation of the slope distribution, while the solid black line inside of the box represents the median. The black dots represent single slope measurements of family members, with the error bars showing the maximum and minimum values.

in contrast to Veritas, which is the youngest and might show younger surfaces. However, it is worth noting that Themis and Hygiea are considerably closer in age than each is to Veritas.

Ursula and Lixiaohua show no unambiguous indication of hydrated members. One difference between the spectra of these families is seen in the fraction of members that exhibit the Turnoff feature. However, the analysis of the Ursula family is based on only four objects and more data are needed to make a proper comparison of this feature between these families.

The sample of families covers a wide range of parental body sizes, from 69 to $492 \mathrm{~km}$. The approach used by Durda et al. (2007) compared the SFD of the families with ones simulated using a combination of smooth-particle hydrodynamics (SPH) and $N$-body codes. Their results provide estimates typically larger than those calculated in this work, with the exception of the Hygiea family. The major differences are found in the case of Themis and Lixiaohua families. In both cases, the higher parental body size suggests that most of the mass is located in the small-sized members, or was detached from the family through dynamical evolution, or lost in the dust produced by the collision. While that could be the case for Themis, it is unlikely that the largest fraction of the mass from the Lixiaohua progenitor was lost, and a smaller parent body size than the one proposed by Durda et al. (2007) is more likely.

Carry (2012) estimated an average bulk density of $1.33 \pm$ $0.58 \mathrm{~g} \mathrm{~cm}^{-3}$ for C-type objects, in agreement with the values we estimated for the Themis and Hygiea families. Carbognani (2017) used a similar methodology to the one described in this work and found a lower limit for the density of C-types of $0.90 \pm 0.05 \mathrm{~g} \mathrm{~cm}^{-3}$. A possible interpretation for this scenario is that there could exist C-type asteroids with lower densities than the members of Themis and Hygiea, which could be explained by variations in grain density (composition) and porosity. For the Ursula family, the low value for density is compatible with the P-/D-types and cometary nuclei (Carry 2012), which is in agreement with the family spectroscopic properties.

The largest members of Hygiea, Themis, and Ursula have been observed in the $3.0 \mu \mathrm{m}$ region. Usui et al. (2019) presented 
the spectrum of (24) Themis obtained by the Akari space telescope, where they identified two features: one at $2.7 \mu \mathrm{m}$ associated with hydrated minerals, and another at around $3.071 \mu \mathrm{m}$ related to water ice. Another indication of water ice as a constituent of the family composition is the presence of three $\mathrm{MBCs}$, in which the cometary activity is suggested to be sublimationdriven (Hsieh et al. 2018). As proposed by Castillo-Rogez \& Schmidt (2010) and Marsset et al. (2016), a possible explanation for the spectral diversity of Themis, which includes hydrated members, MBCs, and surface ice on two family members, is that the Themis parent body could be partially differentiated.

The spectrum of (375) Ursula was observed by Rivkin et al. (2003). At that time the absorption feature was considered to be similar to the one in (1) Ceres. However, as noted by Rivkin et al. (2019), the band would now be classified as similar to the one in (24) Themis, also suggesting the presence of water ice in the family. In contrast with Themis, the Ursula family shows little to no sign of hydrated members. The spectroscopic properties and the low density of the Ursula family suggest that the parent body was formed in a similar region to the P-/D-type asteroids and possible cometary nuclei, beyond the snow line.

In the case of the Hygiea family, the analysis by Takir \& Emery (2012) of (10) Hygiea showed an absorption feature classified as similar to the one in (1) Ceres (Takir \& Emery 2012). However, Rivkin et al. (2019) recently acquired seven additional spectra for the object, three of which were described as "rounded" or "Themis-like", three as "Ceres-like", and one as hydrated ("Sharp" or "Pallas-like"). The Ceres-like absorption feature is interpreted as being due to the presence of ammoniated minerals, while the Sharp or Pallas-like feature is related to the presence of hydrated minerals. Usui et al. (2019) identified two significant features at 2.72 and $3.08 \mu \mathrm{m}$ in the spectrum of (10) Hygiea and a small $3.1 \mu \mathrm{m}$ feature as well as a weaker feature at $2.7 \mu \mathrm{m}$ in the spectrum of (52) Europa. These features are related with hydrated minerals and water ice, respectively. Rivkin et al. (2019) also acquired four spectra of (52) Europa, the secondlargest member of the Hygiea family, and all of these presented rounded or Themis-like features, attributed to water-ice. However, Takir \& Emery (2012) suggested that (52) Europa shows a distinct band shape classified as Europa-like, which could lead to a distinct compositional interpretation. The variability in IR spectra for (10) Hygiea could be attributed to a heterogeneous surface. However, Mothé-Diniz et al. (2001) and Vernazza et al. (2019), using different techniques, did not find evidence of strong surface inhomogeneity for this object. As suggested by Rivkin et al. (2019), additional data are needed to fully characterize the surface of (10) Hygiea.

Carruba (2013) and Rivkin et al. (2014) suggested that (10) Hygiea could be an interloper of the family. However, the object is consistent with the family members at the wavelength regions analyzed in this work. Additional investigations at different wavelengths are necessary to confirm or reject this hypothesis. The large diameter of the family's parent body and the similarity of surface properties between (10) Hygiea and the differentiated body (1) Ceres (Rivkin et al. 2019) suggest that the parent body and/or (10) Hygiea have also undergone differentiation.

On the other hand, Veritas and Lixiaohua do not seem to be differentiated. As discussed in De Prá et al. (2020), the Lixiaohua family shows evidence of water ice through the presence of two MBCs among its members and no trace of aqueous alteration. In contrast, the Veritas family shows a large fraction of hydrated members, which indicates compositional homogeneity. Michel et al. (2011) proposed that the asteroid (490) Veritas, the largest member of the homonymous family, could be an interloper in the family. However, the spectroscopic properties of the asteroid are consistent with a highly homogeneous family.

The sizes of the parent bodies of both the Lixiaohua and Veritas families are below $150 \mathrm{~km}$, which is considerably smaller than the parent bodies of Themis and Hygiea. Combined with their spectroscopic properties, this suggests that these families did not originate from the break-up of differentiated bodies. The differences in spectral properties between these two families suggest that they were formed at different places and/or times in the early Solar System, where the Veritas parent body might have formed sooner and was more exposed to radiogenic heating.

\section{Conclusions}

We present new spectroscopic data for the outer-belt families Themis, Hygiea, and Veritas, and include data available in the literature for the Ursula and Lixiaohua families. Our analyses reveal the diversity of surface properties among the families, where the Themis, Hygiea, and Veritas families present neutral mean slopes in the visible and NIR wavelengths. In contrast, Ursula and Lixiaohua families present reddish mean slopes. The analysis of the albedo reveals that the Lixiaohua is also the darkest among these families. The number of hydrated members varies significantly: $77 \%$ of Veritas members show the $0.7 \mu \mathrm{m}$ feature, while Lixiaohua and Ursula show no evidence of hydration.

In addition to the spectroscopic analysis we also estimated the parental body size of each family and the density based on their rotational properties. The parental body sizes of Themis and Hygiea are the largest among the families, and in combination with the analysis of the distribution of physical properties among their members, suggests that these families could have undergone a partial differentiation process. In contrast, Lixiaohua and Veritas present smaller parent body sizes, and are unlikely to have gone through such processing. The Ursula parent body is of intermediate size, and more data are required to understand the origin of the family. We estimated the density limits for the members of the Themis, Hygiea, and Ursula families, where the first two present densities compatible with C-type asteroids, and the latter with P-type, in agreement with their spectroscopic properties.

The results of the analysis performed in this work suggest a diverse scenario for the formation of the parent bodies of the families studied. The differences between Themis, Hygiea, and Veritas could be related to the initial masses of their progenitors, while Ursula and Lixiaohua could have formed in separate and distinct regions of the protoplanetary disk, and were scattered to their current orbits. However, additional investigations of the compositions and physical properties of their members and further analyses of the remaining primitive outer-belt families are required to test these hypotheses. The continuation of the PRIMASS and future data releases of asteroid spectra from Gaia will greatly contribute to enhancing the analysis of these populations.

Acknowledgements. M.D.P. acknowledges funding from the Prominent Postdoctoral Program of the University of Central Florida. N.P.-A. acknowledges support from the 17-PDART17-2-0097 project funded by the PDART-ROSES 2017 program. J.L. acknowledge support from the AYA2015-67772-R (MINECO, Spain). J.de.L. acknowledges support from MINECO under the 2015 Severo Ochoa Program SEV-2015-0548 and the AYA-2017-89090-P. This work is based in part on observations obtained at the Southern Astrophysical Research Telescope (SOAR), which is a joint project of the Ministério da Ciência, Tecnologia, Inovação e Comunicações (MCTIC) do Brasil, the U.S. National Optical Astronomy Observatory (NOAO), the University of North Carolina at Chapel Hill (UNC), and Michigan State University (MSU). 


\section{References}

Astropy Collaboration (Robitaille, T. P., et al.) 2013, A\&A, 558, A33 Astropy Collaboration (Price-Whelan, A. M., et al.) 2018, AJ, 156, 123 Brož, M., \& Vokrouhlický, D. 2008, MNRAS, 390, 715

Brož, M., Morbidelli, A., Bottke, W. F., et al. 2013, A\&A, 551, A117

Bus, S. J., \& Binzel, R. P. 2002, Icarus, 158, 146

Bus, S., \& Binzel, R. P. 2004, NASA Planetary Data System, EAR

Campins, H., Hargrove, K., Pinilla-Alonso, N., et al. 2010, Nature, 464, 1320

Carbognani, A. 2017, Planet. Space Sci., 147, 1

Carruba, V. 2013, MNRAS, 431, 3557

Carry, B. 2012, Planet. Space Sci., 73, 98

Carvano, J. M., Mothé-Diniz, T., \& Lazzaro, D. 2003, Icarus, 161, 356

Carvano, J. M., Hasselmann, P. H., Lazzaro, D., \& Mothé-Diniz, T. 2010, A\&A, 510, A43

Castillo-Rogez, J. C., \& Schmidt, B. E. 2010, Geophys. Res. Lett., 37, L10202

Cellino, A., Bus, S. J., Doressoundiram, A., \& Lazzaro, D. 2002, Asteroids III (Tucson, AZ: University of Arizona Press), 633

de León, J., Pinilla-Alonso, N., Campins, H., Licand ro, J., \& Marzo, G. A. 2012, Icarus, 218, 196

de León, J., Pinilla-Alonso, N., Delbo, M., et al. 2016, Icarus, 266, 57

DeMeo, F. E., \& Carry, B. 2014, Nature, 505, 629

DeMeo, F. E., Binzel, R. P., Slivan, S. M., \& Bus, S. J. 2009, Icarus, 202, 160

DeMeo, F. E., Alexander, C. M. O., Walsh, K. J., Chapman, C. R., \& Binzel, R. P. 2015, Asteroids IV (Tucson, AZ: University of Arizona Press), 13

De Prá, M. N., Pinilla-Alonso, N., Carvano, J. M., et al. 2018a, Icarus, 311, 35

De Prá, M. N., Carvano, J., Morate, D., Pinilla-Alonso, N., \& Licandro, J. 2018b, AAS/Division for Planetary Sciences Meeting Abstracts, 315.02

De Prá, M., Licandro, J., Pinilla-Alonso, N., et al. 2020, Icarus, 338, 113473

Descamps, P., Marchis, F., Michalowski, T., et al. 2007, Icarus, 187, 482

Durda, D. D., Bottke, W. F., Nesvorný, D., et al. 2007, Icarus, 186, 498

Feierberg, M. A., Lebofsky, L. A., \& Tholen, D. J. 1985, Icarus, 63, 183

Florczak, M., Lazzaro, D., Mothé-Diniz, T., Angeli, C. A., \& Betzler, A. S. 1999, A\&AS, 134, 463

Fornasier, S., Lantz, C., Barucci, M. A., \& Lazzarin, M. 2014, Icarus, 233, 163

Fornasier, S., Lantz, C., Perna, D., et al. 2016, Icarus, 269, 1

Hasselmann, P. H., Carvano, J. M., \& Lazzaro, D. 2011, NASA Planetary Data System, 145

Hendrix, A. R., \& Vilas, F. 2019, Geophys. Res. Lett., 46, 14, 307

Hirayama, K. 1918, AJ, 31, 185

Hsieh, H. H., \& Jewitt, D. 2006, Science, 312, 561

Hsieh, H. H., Jewitt, D. C., \& Fernández, Y. R. 2004, AJ, 127, 2997

Hsieh, H. H., Novaković, B., Kim, Y., \& Brasser, R. 2018, AJ, 155, 96

Hunter, J. D. 2007, Comput. Sci. Eng., 9, 90

Jones, E., Oliphant, T., Peterson, P., et al. 2001, SciPy: Open source scientific tools for Python

Kaluna, H. M., Masiero, J. R., \& Meech, K. J. 2016, Icarus, 264, 62
Landsman, Z. A., Licandro, J., Campins, H., et al. 2016, Icarus, 269, 62

Lazzaro, D., Angeli, C. A., Carvano, J. M., et al. 2007, NASA Planetary Data System, EAR

Licandro, J., Campins, H., Kelley, M., et al. 2011, A\&A, 525, A34

Licandro, J., Hargrove, K., Kelley, M., et al. 2012, A\&A, 537, A73

Mainzer, A. K., Bauer, J. M., Cutri, R. M., et al. 2016, NASA Planetary Data System, 247

Marchis, F., Descamps, P., Berthier, J., et al. 2008, Icarus, 195, 295

Marsset, M., Vernazza, P., Birlan, M., et al. 2016, A\&A, 586, A15

Michel, P., Jutzi, M., Richardson, D. C., \& Benz, W. 2011, Icarus, 211, 535

Morate, D., de León, J., De Prá, M., et al. 2016, A\&A, 586, A129

Morate, D., de León, J., De Prá, M., et al. 2018, A\&A, 610, A25

Morate, D., de León, J., De Prá, M., et al. 2019, A\&A, 630, A141

Morbidelli, A., Chambers, J., Lunine, J. I., et al. 2000, Meteorit. Planet. Sci., 35 1309

Mothé-Diniz, T., di Martino, M., Bendjoya, P., Doressoundiram, A., \& Migliorini, F. 2001, Icarus, 152, 117

Mothé-Diniz, T., Roig, F., \& Carvano, J. M. 2005, Icarus, 174, 54

Nesvorny, D. 2015, NASA Planetary Data System, EAR

Nesvorný, D., Brož, M., \& Carruba, V. 2015, Identification and Dynamical Properties of Asteroid Families, 297

Popescu, M., Licandro, J., Morate, D., et al. 2016, A\&A, 591, A115

Pravec, P., \& Harris, A. W. 2000, Icarus, 148, 12

Pravec, P., \& Harris, A. W. 2007, Icarus, 190, 250

Rivkin, A. S. 2012, Icarus, 221, 744

Rivkin, A. S., \& Emery, J. P. 2010, Nature, 464, 1322

Rivkin, A. S., Davies, J. K., Johnson, J. R., et al. 2003, Meteorit. Planet. Sci., 38, 1383

Rivkin, A. S., Asphaug, E., \& Bottke, W. F. 2014, Icarus, 243, 429

Rivkin, A. S., Campins, H., Emery, J. P., et al. 2015, Asteroids IV (Tucson, AZ: University of Arizona Press), 65

Rivkin, A. S., Howell, E. S., \& Emery, J. P. 2019, J. Geophys. Res. Planets, 124, 1393

Takir, D., \& Emery, J. P. 2012, Icarus, 219, 641

Tanga, P., Cellino, A., Michel, P., et al. 1999, Icarus, 141, 65

Tedesco, E. F. 1994, NASA Planetary Data System

Usui, F., Hasegawa, S., Ootsubo, T., \& Onaka, T. 2019, PASJ, 71, 1

Van Der Walt, S., Colbert, S. C., \& Varoquaux, G. 2011, Comput. Sci. Eng., 13, 22

Vernazza, P., Jorda, L., Ševeček, P., et al. 2019, Nat. Astron., 477

Vilas, F. 1994, Icarus, 111, 456

Vilas, F. 1995, Icarus, 115, 217

Vilas, F., Smith, B. A., McFadden, L. A., et al. 2006, NASA Planetary Data System, EAR

Warner, B. D., Harris, A. W., \& Pravec, P. 2009, Icarus, 202, 134

Xu, S., Binzel, R. P., Burbine, T. H., \& Bus, S. J. 1996, NASA Planetary Data System, EAR

Zappala, V., \& Cellino, A. 1992, Celest. Mech. Dyn. Astron., 54, 207

Ziffer, J., Campins, H., Licandro, J., et al. 2011, Icarus, 213, 538 


\section{Appendix A: Additional figures and tables}

Table A.1. Asteroids observational conditions - visible.

\begin{tabular}{|c|c|c|c|c|c|c|c|c|c|c|}
\hline $\begin{array}{l}\text { Asteroid } \\
\text { number }\end{array}$ & $\begin{array}{l}\text { Asteroid } \\
\text { name }\end{array}$ & $\begin{array}{l}\text { Family } \\
\text { name }\end{array}$ & Date & $\begin{array}{c}\text { Time start } \\
\text { (UT) }\end{array}$ & $\begin{array}{l}\text { Slit } \\
\left({ }^{\prime \prime}\right)\end{array}$ & Airmass & $\begin{array}{c}V \\
(\mathrm{mag})\end{array}$ & $\begin{array}{c}\alpha \\
\left({ }^{\circ}\right)\end{array}$ & $\begin{array}{c}T_{\text {EXP }} \\
(\mathrm{s})\end{array}$ & $\begin{array}{c}\text { Solar } \\
\text { analogs } \\
\end{array}$ \\
\hline 268 & Adorea & Themis & $2012-03-27$ & $07: 55$ & 1.68 & 1.08 & 12.48 & 12.60 & 60.0 & $1,2,3,4$ \\
\hline 561 & Ingwelde & Themis & 2011-02-08 & $01: 44$ & 1.03 & 1.765 & 16.29 & 19.01 & 360.0 & 1 \\
\hline 1581 & Abanderada & Themis & 2011-02-01 & $06: 56$ & 1.03 & 1.263 & 15.53 & 15.52 & 480.0 & 1 \\
\hline 1674 & Groeneveld & Themis & $2011-02-08$ & 02:07 & 1.03 & 1.97 & 16.00 & 18.51 & 360.0 & 1 \\
\hline 1782 & Schneller & Themis & $2012-03-27$ & 06:01 & 1.68 & 1.113 & 15.89 & 4.37 & 420.0 & $1,2,3,4$ \\
\hline 2534 & Houzeau & Themis & 2011-02-01 & $04: 29$ & 1.03 & 1.557 & 16.12 & 2.64 & 320.0 & 1 \\
\hline 2563 & Boyarchuk & Themis & $2011-02-08$ & $05: 23$ & 1.03 & 1.347 & 16.18 & 14.74 & 480.0 & 1 \\
\hline 2918 & Salazar & Themis & 2012-03-29 & $04: 32$ & 1.68 & 1.14 & 16.55 & 1.29 & 480.0 & $1,3,5$ \\
\hline 5429 & 1988 BZ1 & Themis & 2011-01-31 & $06: 14$ & 1.03 & 1.497 & 16.55 & 2.48 & 540.0 & 1 \\
\hline 5594 & Jimmiller & Veritas & $2011-01-31$ & 50 & 1.03 & 1.66 & 16.66 & 5.97 & 400.0 & 1 \\
\hline 6343 & 1993 VK & Veritas & $2012-03-27$ & 48 & 1.68 & 1.48 & 17.83 & 12.06 & 600.0 & $1,2,3,4$ \\
\hline 6374 & Beslan & Veritas & 2011-02-01 & $02: 44$ & 1.03 & 1.36 & 17.06 & 8.33 & 600.0 & 1 \\
\hline 7231 & Porco & Veritas & $2012-03-29$ & 07:07 & 1.68 & 1.2 & 17.86 & 17.97 & 600.0 & $1,3,5$ \\
\hline 9860 & Archaeopteryx & Veritas & $2012-03-29$ & 06:02 & 1.68 & 1.29 & 18.03 & 2.63 & 600.0 & $1,3,5$ \\
\hline 13537 & $1991 \mathrm{SG}$ & Veritas & 2012-03-29 & $08: 21$ & 1.68 & 1.253 & 18.07 & 8.90 & 720.0 & $1,3,5$ \\
\hline 14264 & 2000 AH 142 & Veritas & $2012-03-28$ & 04:01 & 1.68 & 1.18 & 17.90 & 1.81 & 600.0 & $1,4,5$ \\
\hline 24638 & 1981 UC23 & Veritas & $2012-03-28$ & 05:19 & 1.68 & 1.11 & 17.71 & 1.46 & 600.0 & $1,4,5$ \\
\hline 45378 & 2000 AD118 & Veritas & 2012-03-27 & 02:12 & 1.68 & 1.15 & 17.32 & 4.81 & 600.0 & $1,2,3,4$ \\
\hline 1209 & Pumma & Hygiea & $2012-03-27$ & $08: 13$ & 1.68 & 1.14 & 15.29 & 11.73 & 360.0 & $\overline{1,2,3,4}$ \\
\hline 3626 & Ohsaki & Hygiea & $2012-03-27$ & $03: 48$ & 1.68 & 1.313 & 17.39 & 10.46 & 600.0 & $1,2,3,4$ \\
\hline 5116 & Korsor & Hygiea & $2011-02-08$ & $07: 16$ & 1.03 & 1.16 & 17.47 & 14.76 & 540.0 & 1 \\
\hline 5794 & Irmina & Hygiea & 2011-02-01 & $01: 47$ & 1.03 & 1.473 & 17.57 & 12.08 & 600.0 & 1 \\
\hline 6415 & 1993 VR3 & Hygiea & $2012-03-27$ & $04: 46$ & 1.68 & 1.123 & 17.05 & 2.01 & 600.0 & $1,2,3,4$ \\
\hline 1534 & 2000 ED92 & Hygiea & 2011-02-08 & 03:01 & 1.03 & 1.402 & 17.34 & 3.35 & 495.0 & 1 \\
\hline 23,916 & 1998 SD131 & Hygiea & $2012-03-27$ & 03:03 & 1.68 & 1.203 & 17.43 & 0.52 & 600.0 & $1,2,3,4$ \\
\hline 24358 & 2000 AV117 & Hygiea & $2012-03-28$ & $02: 35$ & 1.68 & 1.323 & 17.30 & 4.53 & 600.0 & $1,4,5$ \\
\hline 24956 & 1997 SN10 & Hygiea & $2012-03-29$ & $03: 16$ & 1.68 & 1.192 & 17.86 & 2.16 & 600.0 & $1,3,5$ \\
\hline 25829 & 2000 DU108 & Hygiea & $2012-03-27$ & $08: 44$ & 1.68 & 1.29 & 17.72 & 9.08 & 600.0 & $1,2,3,4$ \\
\hline 26719 & 2001 HQ5 & Hygiea & $2012-03-29$ & $02: 16$ & 1.68 & 1.29 & 17.68 & 2.62 & 600.0 & $1,3,5$ \\
\hline
\end{tabular}

Notes. Solar Analogs: (1) L102-1081, (2) L107-684, (3) L107-998, (4) HD 44594, (5) HD 144584.

Table A.2. Results for the spectroscopic parametrization.

\begin{tabular}{|c|c|c|c|c|c|c|c|c|c|c|c|}
\hline $\begin{array}{l}\text { Asteroid } \\
\text { number }\end{array}$ & Family & $\begin{array}{c}\text { Taxonomic } \\
\text { class }\end{array}$ & $\begin{array}{c}\text { Visible } \\
\text { slope } \\
(\% / 1000 \AA) \\
\end{array}$ & $\begin{array}{c}\text { Visible } \\
\text { slope error } \\
(\% / 1000 \AA)\end{array}$ & $\begin{array}{l}\text { Turn-off } \\
\text { point } \\
(\AA)\end{array}$ & $\begin{array}{c}\text { Turn-off } \\
\text { point error } \\
(\AA)\end{array}$ & $\begin{array}{c}0.7 \mu \mathrm{m} \\
\text { band depth } \\
(\%)\end{array}$ & $\begin{array}{c}0.7 \mu \mathrm{m} \\
\text { band depth } \\
\text { error }(\%)\end{array}$ & $\begin{array}{c}0.7 \mu \mathrm{m} \\
\text { band center } \\
(\AA)\end{array}$ & $\begin{array}{c}0.7 \mu \mathrm{m} \\
\text { band center } \\
\text { error }(\AA)\end{array}$ & Ref \\
\hline \multirow[t]{3}{*}{10} & Hygiea & B & -2.191 & 1.000 & 6087 & 18 & - & - & - & - & 2 \\
\hline & Hygiea & $\mathrm{C}$ & 0.133 & 1.000 & 6010 & 39 & - & - & - & - & 3 \\
\hline & Hygiea & $\mathrm{C}$ & -0.072 & 0.031 & - & - & - & - & - & - & 6 \\
\hline 52 & Hygiea & $\mathrm{Cg}$ & 2.227 & 1.001 & 6039 & 50 & - & - & - & - & 3 \\
\hline \multirow[t]{2}{*}{100} & Hygiea & $\mathrm{S}$ & 7.272 & 1.001 & $*$ & $*$ & - & - & - & - & 3 \\
\hline & Hygiea & A & 11.771 & 0.165 & - & - & - & - & - & - & 6 \\
\hline 108 & Hygiea & $\mathrm{Sv}$ & 8.011 & 1.002 & $*$ & $*$ & - & - & - & - & 3 \\
\hline 159 & Hygiea & $\mathrm{Ch}$ & -0.098 & 1.000 & 5345 & 15 & 2.294 & 0.102 & 6964 & 44 & 3 \\
\hline 538 & Hygiea & B & -0.558 & 0.046 & - & - & - & - & - & - & 6 \\
\hline 867 & Hygiea & B & -3.486 & 0.114 & - & - & - & - & - & - & 6 \\
\hline 1107 & Hygiea & Xk & 2.782 & 1.000 & - & - & - & - & - & - & 3 \\
\hline \multirow[t]{2}{*}{1109} & Hygiea & $\mathrm{D}$ & 7.143 & 1.000 & $*$ & $*$ & - & - & - & - & 4 \\
\hline & Hygiea & $\mathrm{D}$ & 7.197 & 0.106 & - & - & - & - & - & - & 6 \\
\hline
\end{tabular}

Notes. The symbols "-" indicate the spectra that do not show the feature and “*” where the wavelength coverage was not appropriate to evaluate the presence of the feature.

References. (1) This work, (2) SMASS (Xu et al. 1996), (3) SMASSII (Bus \& Binzel 2004), (4) S3OS2 (Lazzaro et al. 2007), (5) Vilas et al. (2006), (6) Mothé-Diniz et al. (2001). 
Table A.2. continued.

\begin{tabular}{|c|c|c|c|c|c|c|c|c|c|c|c|}
\hline $\begin{array}{l}\text { Asteroid } \\
\text { number }\end{array}$ & Family & $\begin{array}{l}\text { Taxonomic } \\
\text { class }\end{array}$ & $\begin{array}{c}\text { Visible } \\
\text { slope } \\
(\% / 1000 \AA)\end{array}$ & $\begin{array}{c}\text { Visible } \\
\text { slope error } \\
(\% / 1000 \AA)\end{array}$ & $\begin{array}{l}\text { Turn-off } \\
\text { point } \\
(\AA)\end{array}$ & $\begin{array}{c}\text { Turn-off } \\
\text { point error } \\
(\AA)\end{array}$ & $\begin{array}{c}0.7 \mu \mathrm{m} \\
\text { band depth } \\
(\%)\end{array}$ & $\begin{array}{c}0.7 \mu \mathrm{m} \\
\text { band depth } \\
\text { error }(\%)\end{array}$ & $\begin{array}{c}0.7 \mu \mathrm{m} \\
\text { band center } \\
(\AA)\end{array}$ & $\begin{array}{c}0.7 \mu \mathrm{m} \\
\text { band center } \\
\text { error }(\AA)\end{array}$ & Ref \\
\hline \multirow[t]{3}{*}{1209} & Hygiea & $X$ & 2.813 & 0.020 & - & - & - & - & - & - & 1 \\
\hline & Hygiea & $\mathrm{T}$ & 6.663 & 1.001 & $*$ & $*$ & - & - & - & - & 4 \\
\hline & Hygiea & $\mathrm{T}$ & 6.367 & 0.208 & - & - & - & - & - & - & 6 \\
\hline 1599 & Hygiea & $S$ & 0.618 & 0.199 & - & - & - & - & - & - & 6 \\
\hline 2436 & Hygiea & B & -3.540 & 0.231 & - & - & - & - & - & - & 6 \\
\hline 3626 & Hygiea & B & -1.470 & 0.027 & 5212 & 30 & - & - & - & - & 1 \\
\hline 4667 & Hygiea & B & -4.554 & 0.108 & - & - & - & - & - & - & 6 \\
\hline 4955 & Hygiea & B & -3.420 & 1.000 & $*$ & $*$ & - & - & - & - & 4 \\
\hline 5116 & Hygiea & $\mathrm{C}$ & 2.934 & 0.022 & 5297 & 12 & - & - & - & - & 1 \\
\hline 5155 & Hygiea & $\mathrm{C}$ & -0.666 & 0.079 & - & - & - & - & - & - & 6 \\
\hline 5794 & Hygiea & B & -6.217 & 0.065 & - & - & - & - & - & - & 1 \\
\hline 6415 & Hygiea & B & -1.050 & 0.021 & 5125 & 21 & - & - & - & - & 1 \\
\hline 15134 & Hygiea & B & -1.686 & 0.047 & - & - & - & - & - & - & 1 \\
\hline 23916 & Hygiea & $\mathrm{C}$ & 0.297 & 0.023 & 5143 & 7 & 3.148 & 0.095 & 7119 & 21 & 1 \\
\hline 24358 & Hygiea & $\mathrm{Cb}$ & 1.118 & 0.024 & 5013 & 22 & 2.361 & 0.095 & 7058 & 34 & 1 \\
\hline 24956 & Hygiea & $\mathrm{B}$ & -1.476 & 0.027 & 4967 & 20 & - & - & - & - & 1 \\
\hline 25829 & Hygiea & $\mathrm{C}$ & 0.304 & 0.032 & 5175 & 21 & - & - & - & - & 1 \\
\hline 26719 & Hygiea & $\mathrm{Xc}$ & 4.151 & 0.031 & - & - & - & - & - & - & 1 \\
\hline \multirow[t]{2}{*}{24} & Themis & B & -0.852 & 0.021 & - & - & - & - & - & - & 3 \\
\hline & Themis & B & -0.808 & 0.022 & $*$ & $*$ & - & - & - & - & 4 \\
\hline \multirow[t]{2}{*}{62} & Themis & B & -1.790 & 0.037 & 5432 & 37 & 1.062 & 0.088 & 6741 & 50 & 3 \\
\hline & Themis & B & -2.074 & 0.014 & $*$ & $*$ & - & - & 6741 & 50 & 4 \\
\hline \multirow[t]{2}{*}{90} & Themis & $\mathrm{Ch}$ & -0.452 & 0.015 & $*$ & $*$ & 1.31 & 0.055 & 7087 & 28 & 4 \\
\hline & Themis & $\mathrm{C}$ & 0.409 & 0.024 & 6735 & 70 & - & - & 7087 & 28 & 3 \\
\hline \multirow[t]{2}{*}{171} & Themis & $\mathrm{C}$ & 0.269 & 0.017 & $*$ & $*$ & - & - & - & - & 4 \\
\hline & Themis & $\mathrm{C}$ & 0.666 & 0.022 & 5986 & 38 & - & - & - & - & 3 \\
\hline 223 & Themis & $\mathrm{Cb}$ & 2.015 & 0.017 & $*$ & $*$ & - & - & - & - & 4 \\
\hline \multirow[t]{2}{*}{268} & Themis & $\mathrm{Cb}$ & 1.519 & 0.017 & $*$ & $*$ & - & - & - & - & 4 \\
\hline & Themis & $\mathrm{C}$ & 1.567 & 0.022 & - & - & - & - & - & - & 1 \\
\hline 316 & Themis & $\mathrm{C}$ & -0.195 & 0.025 & $*$ & $*$ & - & - & - & - & 4 \\
\hline 379 & Themis & $\mathrm{C}$ & 0.142 & 0.020 & 5728 & 39 & - & - & - & - & 3 \\
\hline 383 & Themis & $\mathrm{B}$ & -1.918 & 0.055 & 5319 & 36 & - & - & - & - & 3 \\
\hline 461 & Themis & $X$ & 3.837 & 0.025 & $*$ & $*$ & - & - & - & - & 4 \\
\hline 468 & Themis & $\mathrm{C}$ & 2.785 & 0.050 & $*$ & $*$ & - & - & - & - & 4 \\
\hline 515 & Themis & $\mathrm{Cb}$ & 0.758 & 0.065 & - & - & - & - & - & - & 3 \\
\hline 526 & Themis & $\mathrm{Cb}$ & 0.336 & 0.020 & $*$ & $*$ & - & - & - & - & 4 \\
\hline 561 & Themis & $\mathrm{Ch}$ & 0.304 & 0.020 & 5219 & 29 & - & - & - & - & 1 \\
\hline 621 & Themis & $\mathrm{B}$ & -0.905 & 0.034 & $*$ & $*$ & - & - & - & - & 4 \\
\hline 767 & Themis & B & -2.318 & 0.031 & - & - & - & - & - & - & 3 \\
\hline 846 & Themis & $\mathrm{Cb}$ & 0.912 & 0.026 & $*$ & $*$ & - & - & - & - & 4 \\
\hline 848 & Themis & $\mathrm{Cb}$ & 0.619 & 0.019 & $*$ & $*$ & - & - & - & - & 4 \\
\hline 936 & Themis & B & -1.363 & 0.014 & $*$ & $*$ & - & - & - & - & 4 \\
\hline 954 & Themis & $\mathrm{Cb}$ & 0.783 & 0.028 & $*$ & $*$ & - & - & - & - & 4 \\
\hline 981 & Themis & $\mathrm{C}$ & -0.002 & 0.018 & $*$ & $*$ & - & - & - & - & 4 \\
\hline 1003 & Themis & B & -1.980 & 0.040 & $*$ & $*$ & - & - & - & - & 4 \\
\hline 1229 & Themis & $\mathrm{B}$ & -1.779 & 0.040 & $*$ & $*$ & - & - & - & - & 4 \\
\hline 1302 & Themis & B & -4.068 & 0.020 & $*$ & $*$ & 2.497 & 0.067 & 6596 & 54 & 2 \\
\hline 1340 & Themis & $\mathrm{Ch}$ & -0.974 & 0.018 & $*$ & $*$ & 1.448 & 0.055 & 7084 & 126 & 4 \\
\hline 1487 & Themis & B & -1.431 & 0.021 & $*$ & $*$ & - & - & - & - & 4 \\
\hline \multirow[t]{2}{*}{1539} & Themis & B & -1.726 & 0.027 & 5356 & 32 & - & - & - & - & 3 \\
\hline & Themis & B & -0.612 & 0.016 & $*$ & $*$ & - & - & - & - & 4 \\
\hline 1576 & Themis & B & -1.713 & 0.014 & $*$ & $*$ & - & - & - & - & 4 \\
\hline 1615 & Themis & B & -1.198 & 0.014 & $*$ & $*$ & - & - & - & - & 4 \\
\hline 1674 & Themis & $\mathrm{C}$ & 2.061 & 0.027 & - & - & - & - & - & - & 1 \\
\hline 1691 & Themis & $\mathrm{Cb}$ & 0.396 & 0.022 & $*$ & $*$ & - & - & - & - & 4 \\
\hline 1782 & Themis & B & -2.655 & 0.023 & 5013 & 16 & - & - & - & - & 1 \\
\hline 2296 & Themis & $\mathrm{Cb}$ & 0.328 & 0.023 & $*$ & $*$ & - & - & - & - & 4 \\
\hline 2489 & Themis & $\mathrm{Cb}$ & 0.608 & 0.017 & $*$ & $*$ & 1.343 & 0.053 & 6531 & 53 & 4 \\
\hline 2519 & Themis & $\mathrm{B}$ & -1.495 & 0.028 & $*$ & $*$ & - & - & - & - & 4 \\
\hline
\end{tabular}


M. N. De Prá et al.: A comparative analysis of the outer-belt primitive families

Table A.2. continued.

\begin{tabular}{|c|c|c|c|c|c|c|c|c|c|c|c|}
\hline $\begin{array}{l}\text { Asteroid } \\
\text { number }\end{array}$ & Family & $\begin{array}{l}\text { Taxonomic } \\
\text { class }\end{array}$ & $\begin{array}{c}\text { Visible } \\
\text { slope } \\
(\% / 1000 \AA)\end{array}$ & $\begin{array}{c}\text { Visible } \\
\text { slope error } \\
(\% / 1000 \AA)\end{array}$ & $\begin{array}{l}\text { Turn-off } \\
\text { point } \\
(\AA)\end{array}$ & $\begin{array}{c}\text { Turn-off } \\
\text { point error } \\
(\AA)\end{array}$ & $\begin{array}{c}0.7 \mu \mathrm{m} \\
\text { band depth } \\
(\%)\end{array}$ & $\begin{array}{c}0.7 \mu \mathrm{m} \\
\text { band depth } \\
\text { error }(\%)\end{array}$ & $\begin{array}{c}0.7 \mu \mathrm{m} \\
\text { band center } \\
(\AA)\end{array}$ & $\begin{array}{c}0.7 \mu \mathrm{m} \\
\text { band center } \\
\text { error }(\AA)\end{array}$ & Ref \\
\hline 2524 & Themis & $\mathrm{Ch}$ & -0.765 & 0.015 & * & $*$ & - & - & - & - & 4 \\
\hline 2525 & Themis & $\mathrm{Ch}$ & -0.760 & 0.017 & $*$ & $*$ & 1.432 & 0.059 & 6848 & 43 & 4 \\
\hline 2534 & Themis & $\mathrm{C}$ & 1.340 & 0.033 & 5241 & 42 & - & - & - & - & 1 \\
\hline 2563 & Themis & B & -3.897 & 0.020 & - & - & 2.994 & 0.075 & 6804 & 28 & 1 \\
\hline 2659 & Themis & B & -1.276 & 0.026 & 5633 & 35 & - & - & - & - & 3 \\
\hline 2708 & Themis & B & -2.543 & 0.088 & - & - & - & - & - & - & 3 \\
\hline 2918 & Themis & B & -2.225 & 0.016 & 4977 & 12 & 1.444 & 0.055 & 6963 & 44 & 1 \\
\hline 3128 & Themis & $\mathrm{Cb}$ & 0.280 & 0.023 & $*$ & $*$ & - & - & - & - & 4 \\
\hline 3179 & Themis & $\mathrm{Ch}$ & 0.811 & 0.215 & - & - & - & - & - & - & 3 \\
\hline 3274 & Themis & $\mathrm{C}$ & 0.478 & 0.032 & $*$ & $*$ & - & - & - & - & 4 \\
\hline \multirow[t]{2}{*}{3507} & Themis & B & -0.978 & 0.019 & $*$ & $*$ & - & - & - & - & 4 \\
\hline & Themis & $\mathrm{Ch}$ & 0.423 & 0.127 & - & - & - & - & - & - & 3 \\
\hline 3615 & Themis & $\mathrm{Cb}$ & 0.810 & 0.024 & $*$ & $*$ & - & - & - & - & 4 \\
\hline 3663 & Themis & $\mathrm{C}$ & 0.067 & 0.026 & $*$ & $*$ & - & - & - & - & 4 \\
\hline 3832 & Themis & $\mathrm{Cb}$ & 0.547 & 0.028 & $*$ & $*$ & - & - & - & - & 4 \\
\hline 4143 & Themis & $\mathrm{Cb}$ & 0.326 & 0.023 & $*$ & $*$ & - & - & - & - & 4 \\
\hline 4759 & Themis & $\mathrm{Ch}$ & -0.293 & 0.027 & $*$ & $*$ & - & - & - & - & 4 \\
\hline 4778 & Themis & $X$ & 3.630 & 0.047 & $*$ & $*$ & - & - & - & - & 4 \\
\hline 5045 & Themis & $\mathrm{Cb}$ & 0.100 & 0.034 & $*$ & $*$ & - & - & - & - & 4 \\
\hline 5429 & Themis & $\mathrm{B}$ & -3.854 & 0.063 & - & - & - & - & - & - & 1 \\
\hline 6297 & Themis & B & -0.147 & 0.031 & $*$ & $*$ & - & - & - & - & 4 \\
\hline 8518 & Themis & $\mathrm{Ch}$ & -0.742 & 0.024 & $*$ & $*$ & - & - & - & - & 4 \\
\hline 8906 & Themis & $\mathrm{Ch}$ & -0.928 & 0.059 & $*$ & $*$ & - & - & - & - & 4 \\
\hline 490 & Veritas & $\mathrm{Cgh}$ & 1.745 & 0.024 & 5434 & 18 & 1.036 & 0.073 & 6902 & 38 & 3 \\
\hline \multirow[t]{2}{*}{1086} & Veritas & $\mathrm{Ch}$ & 0.081 & 0.022 & $*$ & $*$ & 4.424 & 0.073 & 7059 & 42 & 4 \\
\hline & Veritas & $\mathrm{Ch}$ & -0.248 & 0.051 & 5262 & 21 & 2.195 & 0.13 & 7059 & 42 & 3 \\
\hline 2147 & Veritas & $\mathrm{Ch}$ & 1.213 & 0.106 & 5421 & 57 & 3.04 & 0.233 & 7276 & 57 & 3 \\
\hline 2428 & Veritas & $\mathrm{Ch}$ & 0.039 & 0.099 & 5459 & 32 & 2.4 & 0.309 & 6959 & 77 & 3 \\
\hline 2934 & Veritas & $\mathrm{B}$ & -1.175 & 0.128 & - & - & 3.634 & 0.448 & 6984 & 119 & 3 \\
\hline 3090 & Veritas & $\mathrm{Cg}$ & 2.568 & 0.154 & 5738 & 50 & - & - & - & - & 3 \\
\hline 3542 & Veritas & $\mathrm{B}$ & -0.462 & 0.152 & - & - & - & - & - & - & 3 \\
\hline 5592 & Veritas & $X$ & 2.792 & 0.040 & $*$ & $*$ & 3.32 & 0.095 & 7046 & 16 & 4 \\
\hline 5594 & Veritas & $\mathrm{Ch}$ & 1.384 & 0.048 & 5558 & 20 & - & - & - & - & 1 \\
\hline 6343 & Veritas & $\mathrm{C}$ & 2.355 & 0.035 & 5258 & 13 & - & - & - & - & 1 \\
\hline 6374 & Veritas & $\mathrm{Ch}$ & 0.789 & 0.033 & 5201 & 18 & 2.99 & 0.117 & 7023 & 44 & 1 \\
\hline 7231 & Veritas & $\mathrm{C}$ & 2.256 & 0.037 & 5222 & 17 & 3.351 & 0.134 & 7086 & 32 & 1 \\
\hline 9860 & Veritas & B & -1.464 & 0.031 & 5030 & 12 & 3.32 & 0.122 & 7028 & 28 & 1 \\
\hline 13537 & Veritas & $\mathrm{Ch}$ & 0.299 & 0.040 & 5284 & 19 & 1.74 & 0.1450 & 7159 & 49 & 1 \\
\hline 14264 & Veritas & $\mathrm{Ch}$ & 1.582 & 0.026 & 5277 & 7 & 2.784 & 0.095 & 7056 & 23 & 1 \\
\hline 24638 & Veritas & $\mathrm{Ch}$ & 0.144 & 0.025 & 5228 & 11 & 4.041 & 0.1 & 6992 & 21 & 1 \\
\hline 45378 & Veritas & $\mathrm{Cgh}$ & 2.723 & 0.022 & 5241 & 9 & 1.567 & 0.078 & 7032 & 25 & 1 \\
\hline \multirow[t]{2}{*}{375} & Ursula & $X$ & 3.432 & 0.022 & $*$ & $*$ & - & - & - & - & 5 \\
\hline & Ursula & $\mathrm{Xk}$ & 3.175 & 0.048 & 6164 & 48 & - & - & - & - & 3 \\
\hline \multirow[t]{2}{*}{601} & Ursula & $\mathrm{C}$ & 1.304 & 0.076 & 6267 & 16 & - & - & - & - & 3 \\
\hline & Ursula & $\mathrm{C}$ & 0.215 & 0.015 & $*$ & $*$ & - & - & - & - & 4 \\
\hline 618 & Ursula & $\mathrm{Cb}$ & 1.558 & 0.012 & $*$ & $*$ & - & - & - & - & 4 \\
\hline \multirow[t]{2}{*}{973} & Ursula & $X$ & 3.380 & 0.032 & $*$ & $*$ & - & - & - & - & 4 \\
\hline & Ursula & $\mathrm{Xk}$ & 4.208 & 0.067 & 5892 & 80 & - & - & - & - & 3 \\
\hline 1306 & Ursula & $\mathrm{T}$ & 5.510 & 0.030 & $*$ & $*$ & - & - & - & - & 4 \\
\hline 1520 & Ursula & $\mathrm{Cg}$ & 1.979 & 0.069 & 5848 & 130 & - & - & - & - & 3 \\
\hline 5959 & Ursula & $X$ & 2.368 & 0.035 & $*$ & $*$ & - & - & - & - & 4 \\
\hline
\end{tabular}


A\&A 643, A102 (2020)

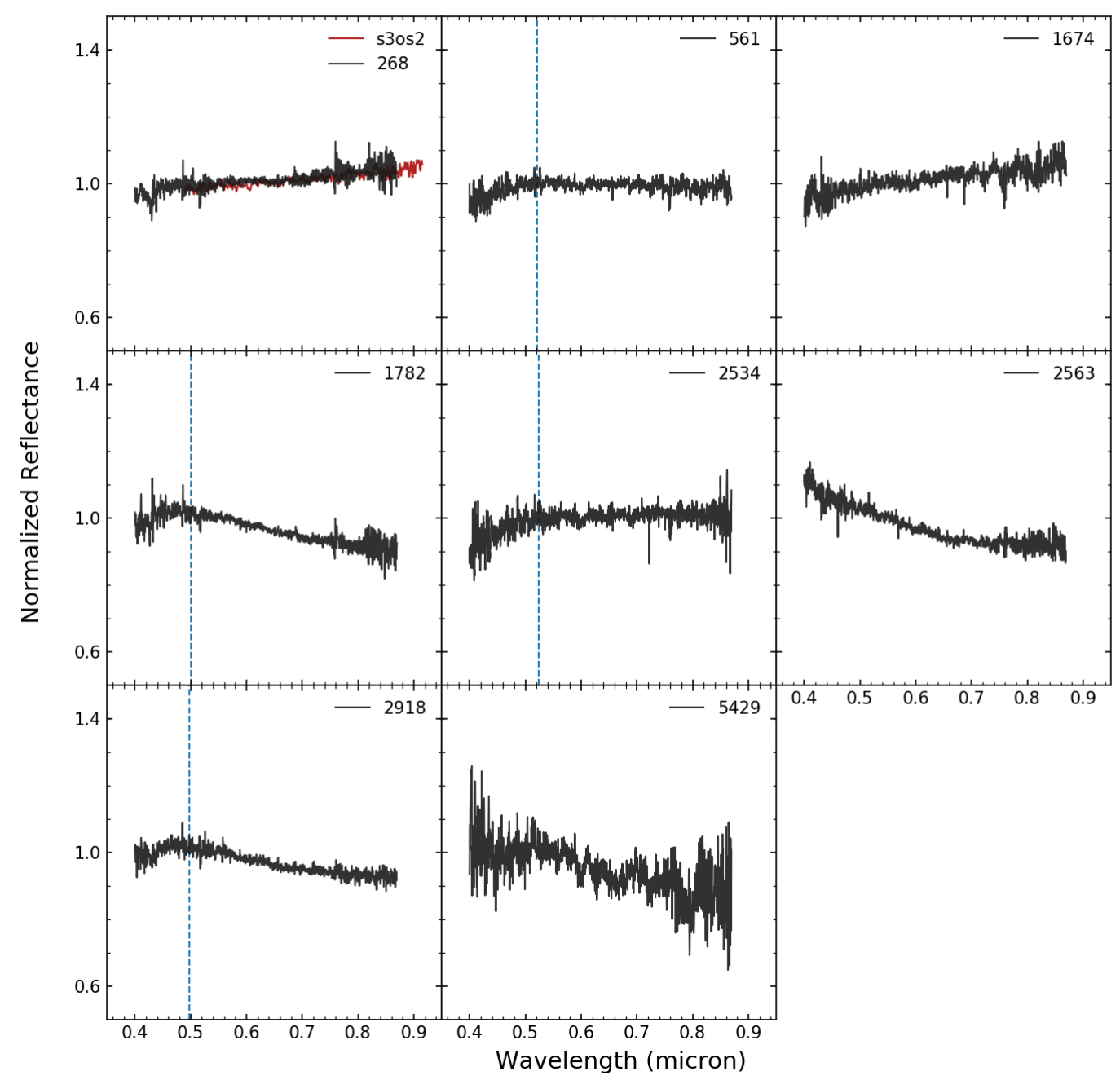

Fig. A.1. Visible spectroscopy of asteroids from the Themis family. Spectra of the same objects that were observed by different works are shown in red. The blue lines represent the turn-off point. 
M. N. De Prá et al.: A comparative analysis of the outer-belt primitive families

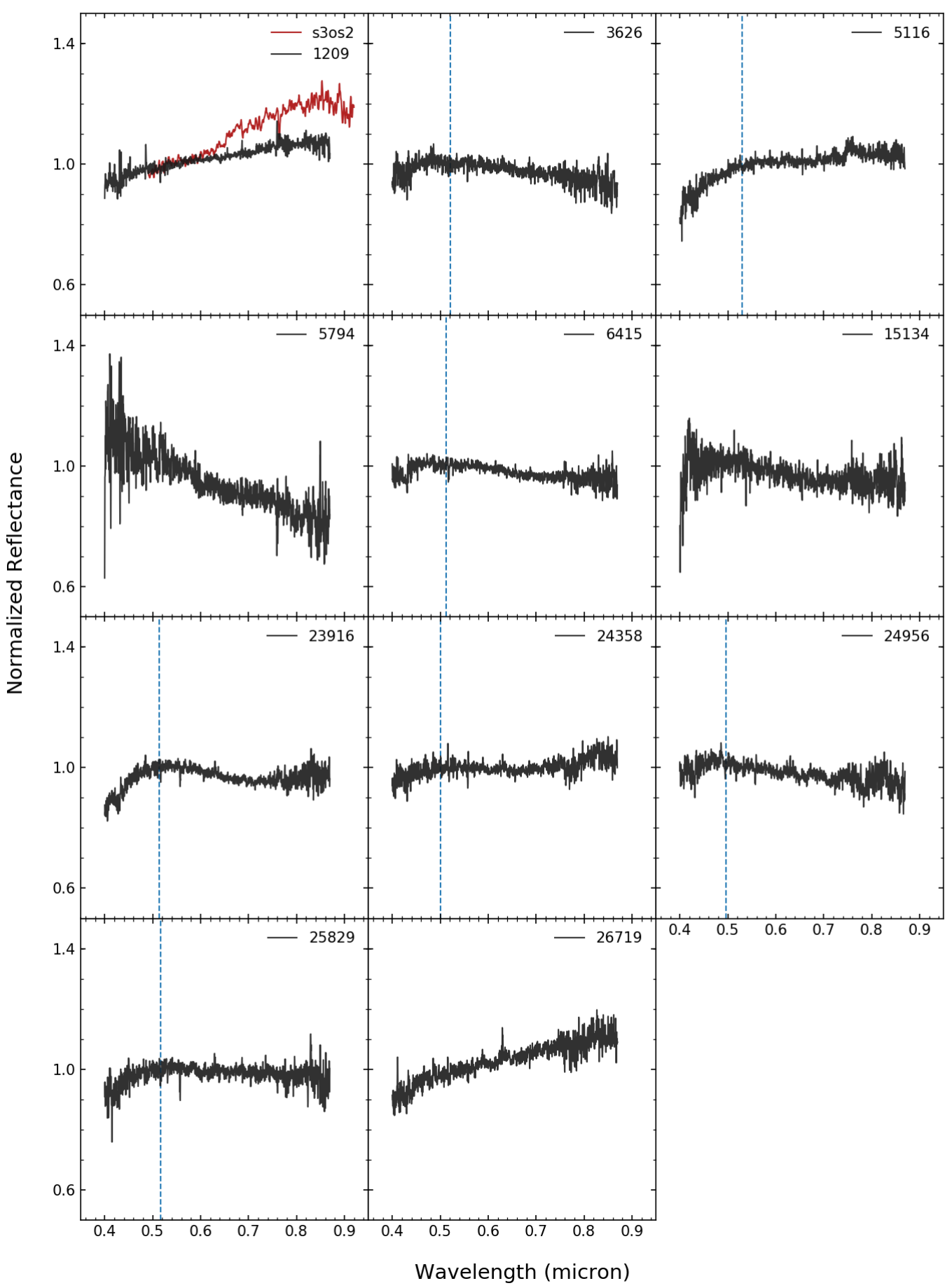

Fig. A.2. Visible spectroscopy of asteroids from the Hygiea family. Spectra of the same objects that were observed by different works are shown in red. The blue lines represent the turn-off point. 
A\&A 643, A102 (2020)

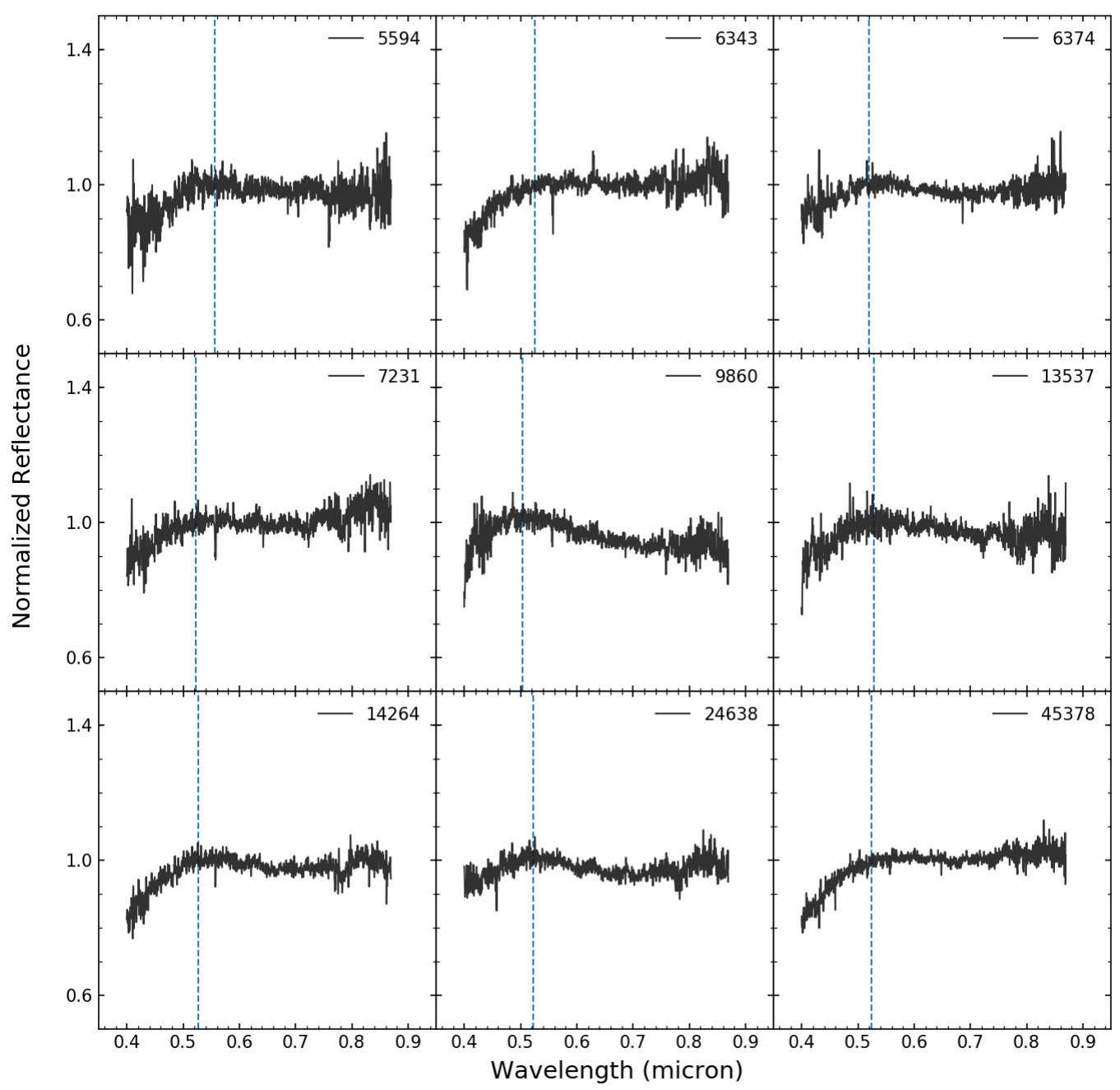

Fig. A.3. Visible spectroscopy of asteroids from the Veritas family. The blue lines represent the turn-off point. 